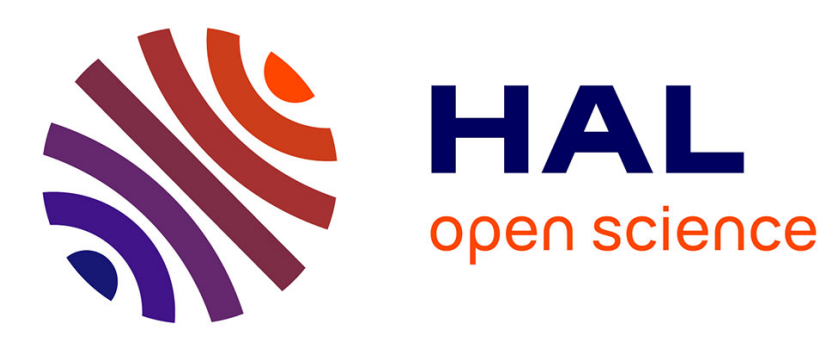

\title{
Cocks-Pinch curves of embedding degrees five to eight and optimal ate pairing computation
}

\author{
Aurore Guillevic, Simon Masson, Emmanuel Thomé
}

\section{To cite this version:}

Aurore Guillevic, Simon Masson, Emmanuel Thomé. Cocks-Pinch curves of embedding degrees five to eight and optimal ate pairing computation. Designs, Codes and Cryptography, 2020, 88 (6), pp.10471081. 10.1007/s10623-020-00727-w . hal-02305051v2

\section{HAL Id: hal-02305051 \\ https://hal.inria.fr/hal-02305051v2}

Submitted on 9 Mar 2020

HAL is a multi-disciplinary open access archive for the deposit and dissemination of scientific research documents, whether they are published or not. The documents may come from teaching and research institutions in France or abroad, or from public or private research centers.
L'archive ouverte pluridisciplinaire HAL, est destinée au dépôt et à la diffusion de documents scientifiques de niveau recherche, publiés ou non, émanant des établissements d'enseignement et de recherche français ou étrangers, des laboratoires publics ou privés. 


\title{
Cocks-Pinch curves of embedding degrees five to eight and optimal ate pairing computation
}

\author{
Aurore Guillevic ${ }^{1}$, Simon Masson ${ }^{1,2}$, and Emmanuel Thomé ${ }^{1}$ \\ 1 Université de Lorraine, CNRS, Inria, Nancy, France \\ 2 Thales, Gennevilliers, France
}

\begin{abstract}
Recent algorithmic improvements of discrete logarithm computation in special extension fields threaten the security of pairing-friendly curves used in practice. A possible answer to this delicate situation is to propose alternative curves that are immune to these attacks, without compromising the efficiency of the pairing computation too much. We follow this direction, and focus on embedding degrees 5 to 8; we extend the CocksPinch algorithm to obtain pairing-friendly curves with an efficient ate pairing. We carefully select our curve parameters so as to thwart possible attacks by "special" or "tower" Number Field Sieve algorithms. We target a 128-bit security level, and back this security claim by time estimates for the DLP computation. We also compare the efficiency of the optimal ate pairing computation on these curves to $k=12$ curves (Barreto-Naehrig, Barreto-Lynn-Scott), $k=16$ curves (Kachisa-Schaefer-Scott) and $k=1$ curves (Chatterjee-Menezes-Rodríguez-Henríquez).
\end{abstract}

\section{Introduction}

Constructive pairings were introduced into cryptography in the early 2000's with a one round tripartite Diffie-Hellman key exchange [31], identity-based encryption [12], and short signatures [13]. More recently, new applications have been proposed, e.g. zero-knowledge proofs [11] used in the Zcash cryptocurrency and electronic voting.

Pairing-based cryptography relies on the hardness of the discrete logarithm (DL) problem over two groups: an elliptic curve $E\left(\mathbb{F}_{p}\right)$ and a finite field $\mathbb{F}_{p^{k}}, k$ being the embedding degree. Advances on discrete logarithm computation over special field extensions $\mathbb{F}_{p^{k}}$ force us to review not only the parameter sizes of curves used in practice, but also the families of curves used to generate parameters.

The computation of discrete logarithms in finite fields and the factorisation of large integers are both addressed by the Number Field Sieve algorithm (NFS). Its complexity is $L_{p^{k}}(1 / 3, c+o(1))$, with the notation $L_{p^{k}}(\alpha, c)=$ $\exp \left(c\left(\log p^{k}\right)^{\alpha}\left(\log \log p^{k}\right)^{1-\alpha}\right)$. For the families of finite fields that we consider in this paper, we have $1.526 \leq c \leq 2.20$. The value of $c$ depends on the variant of the NFS algorithm. For prime fields $\mathbb{F}_{p}, c=1.923$ and this complexity was considered as the reference for choosing the size of any finite field $\mathbb{F}_{p^{k}}$, where $p$ is medium to large compared to $p^{k}$. However, for some degrees $k$ the NFS algorithm can be parameterised differently, yielding a better complexity with a 
smaller $c$. The size of the finite field must then be increased so as to maintain the same security as previously thought, that is with $c=1.923$. In the context of finite fields (unrelated to pairings), so-called special primes $p$ are subject to the special NFS (SNFS) variant with $c=1.526$ : in these cases, the size of $p$ gives a false sense of security $[28,53,49,47,27]$. The most efficient pairings were obtained with specific families of pairing-friendly curves, where the prime $p$ is special (see Table 2). In 2013, Joux and Pierrot exploited this weakness [33]. In 2015, Barbulescu, Gaudry and Kleinjung revisited Schirokauer's Tower NFS (TNFS) variant, and obtained $c=1.526$ in a theoretical "special Tower NFS" variant (STNFS) [9]. In 2016, Kim and Barbulescu applied the STNFS variant to finite fields of composite extension degree $k$ and obtained, in the best case, an algorithm of complexity with $c=1.526$ [36]. This means that, asymptotically, the size of the finite field $\mathbb{F}_{p^{k}}$ should be roughly doubled to provide the same security as thought before.

However, the asymptotic complexity does not provide enough accuracy to deduce the sizes of the finite fields that we would like to use for cryptography today. Menezes, Sarkar and Singh already showed that the efficiency of the STNFS variants depends on the total size and the extension degree [42], and that the STNFS variant with the best asymptotic complexity is not necessarily the best variant that applies to a 3000-bit finite field. Then Barbulescu and Duquesne proposed a way to refine the estimates [7] and proposed parameters for 128 bits of security for BN, BLS, and KSS curves (where $k=12,16$ and 18).

The rule of thumb that prime fields of 3072 to 3200 bits offer 128 bits of security is extrapolated from the asymptotic complexity, re-scaled according to a record computation. It almost systematically relies on the bodacious assumption $o(1)=0$ in the asymptotic formula. Since a record computation is not available for the TNFS and STNFS algorithms, the papers [42,7] simulate a simplified version of the new algorithms to estimate their costs. We recall in Table 1 the popular pairing-friendly curves before the STNFS algorithm (2015), and the new propositions of [7], together with our estimate of the running-time of a DL computation in the corresponding fields $\mathbb{F}_{p^{k}}$.

\begin{tabular}{lccccc|ccc}
\multicolumn{7}{c|}{ parameters } & \multicolumn{3}{c}{ Cost of DL computation } \\
\hline curve & $\log _{2} p$ & $\log _{2} r$ & $k$ & $\log _{2} p^{k}$ & $\operatorname{deg} p(u)$ & $\begin{array}{c}\text { DL on } E\left(\mathbb{F}_{p}\right) \\
(\approx \sqrt{r})\end{array}$ & $\begin{array}{c}\text { STNFS on } \mathbb{F}_{p^{k}} \\
(7]\end{array}$ & $\S \mathrm{B}$ \\
BN & 254 & 254 & 12 & 3039 & 4 & $2^{127}$ & $2^{100}$ & $\mathbf{2}^{\mathbf{1 0 3}}$ \\
BN & 446 & 446 & 12 & 5343 & 4 & $2^{223}$ & - & $\mathbf{2}^{\mathbf{1 3 2}}$ \\
BN & 462 & 462 & 12 & 5535 & 4 & $2^{231}$ & $2^{131}$ & $\mathbf{2}^{\mathbf{1 3 4}}$ \\
BLS12 & 381 & 255 & 12 & 4572 & 6 & $2^{127}$ & - & $\mathbf{2}^{\mathbf{1 2 6}}$ \\
BLS12 & 461 & 308 & 12 & 5525 & 6 & $2^{154}$ & $2^{132}$ & $\mathbf{2}^{\mathbf{1 3 4}}$ \\
KSS16 & 330 & 257 & 16 & 5280 & 10 & $2^{128}$ & $2^{139}$ & $\mathbf{2}^{\mathbf{1 4 1}}$ \\
KSS16 & 339 & 263 & 16 & 5411 & 10 & $2^{131}$ & $2^{139}$ & $\mathbf{2}^{\mathbf{1 4 1}}$
\end{tabular}

Table 1: Sizes and DL cost estimates. 


\begin{tabular}{l}
$\overline{\text { BN, } \rho=1, p=36 u^{4}+36 u^{3}+24 u^{2}+6 u+1}$ \\
\hline BN-254 $\quad u=-2^{62}-2^{55}-1(2010)[45]$ \\
BN-446 $\quad u=2^{110}+2^{36}+1(2010)[45]$ \\
BN-462 $\quad u=2^{114}+2^{101}-2^{14}-1(2018)[7]$ \\
\hline$\overline{B L S 12, \rho=3 / 2, p=\left(u^{6}-2 u^{5}+2 u^{3}+u+1\right) / 3}$ \\
\hline BLS12-381 $u=-\left(2^{63}+2^{62}+2^{60}+2^{57}+2^{48}+2^{16}\right)(2017$ Zcash [15] $)$ \\
BLS12-461 $u=-2^{77}+2^{50}+2^{33}(2018)[7]$ \\
\hline \hline KSS16, $\rho=5 / 4$ \\
$p=\left(u^{10}+2 u^{9}+5 u^{8}+48 u^{6}+152 u^{5}+240 u^{4}+625 u^{2}+2398 u+3125\right) / 980$ \\
\hline KSS16-330 $u=-2^{34}+2^{27}-2^{23}+2^{20}-2^{11}+1(2018)[7]$ \\
KSS16-339u= $u=2^{35}-2^{32}-2^{18}+2^{8}+1(2018)[7]$ \\
\hline \hline
\end{tabular}

Table 2: Parameters of commonly used pairing-friendly curves

Generation of pairing-friendly curves. Pairings on elliptic curves map pairs in $E\left(\mathbb{F}_{p}\right)[r] \times E\left(\mathbb{F}_{p^{k}}\right)[r]$ to $\mathbb{F}_{p^{k}}$, and the embedding degree $k$ should be small, say $1 \leq k \leq 20$. The first suggested pairings used supersingular curves, because they were the first known way to produce curves with a small embedding degree. Then two other approaches were proposed (and surveyed in [26]). On the one hand, polynomial methods parameterise the characteristic $p$, the trace $t$, and the curve order $r$ by polynomials. The parameterisation enables two fast variants of the Tate pairing: the ate or optimal ate pairing [52]. A Tate pairing computation contains an internal loop (the Miller loop) of length $\log _{2} r$, while this length is $\log _{2}(t-1)\left(\right.$ resp. $\left.\left(\log _{2} r\right) / \varphi(k)\right)$ for an ate (resp. optimal ate) pairing. On the other hand, non-polynomial methods were also proposed. Because of the large gap in pairing efficiency, the latter methods attracted less attention, and were not optimised.

Pairing-friendly curves from polynomial constructions enjoy fast implementations (e.g., [2]). However, the downside is that since $p$ is parameterised by a polynomial, the Kim-Barbulescu STNFS algorithm applies (at least in some cases), and security estimates need to be revised. Alternatively, cryptographers look for new pairing-friendly curves. Chatterjee, Menezes and Rodríguez-Henríquez propose in [16] to revisit curves of degree one (and trace $t=2$ ), avoiding the TNFS attack. The target finite field is a prime field so it only requires $\log _{2}(p) \geq 3072$ to get 128-bits of security [44]. Fotiadis and Konstantinou propose in [24] families of elliptic curves from a variant of the (polynomial) Brezing-Weng method. For composite embedding degree, they increase the parameter sizes in order to get TNFS-resistant curves, but they also propose curves of prime embedding degree for which the TNFS attack is restricted to $\operatorname{deg} h=k$ only. Unfortunately, the prime $p$ has a polynomial form with tiny coefficients so the special variants (SNFS and STNFS) still apply.

Our contribution. In this article, we take a different approach: to avoid having to increase the size of the target finite field $\mathbb{F}_{p^{k}}$, we choose a Cocks-Pinch curve so that $p$ is not special and the special variants (SNFS, STNFS) do not apply. 
But on Cocks-Pinch curves, no optimal ate pairing is available, and the ate pairing is as slow as the Tate pairing because of a large trace $t$. So we modify the Cocks-Pinch method to obtain a trace of smallest possible size $\left(\log _{2} r\right) / \varphi(k)$, and arrange so that the ate pairing or its variant $[52, \S 2.2]$ is available. We obtain an optimal pairing as defined by Vercauteren in [52]. We generate curves of embedding degree 5 to 8 in order to compare the efficiency of the ate pairing with different sizes of parameters.

- For composite extension degrees $(k=6$ and 8$)$, we reuse some of the optimisations from the literature to obtain a pairing computation that is as efficient as with competing constructions. While it is true that by doing so, we endow our prime $p$ with some special structure, we argue in this paper that the multivariate nature of our parameterisation offers much more flexibility than known constructions, and to our knowledge thwarts all known "special" variants of NFS.

- For prime embedding degrees $(k=5$ and 7$)$, the TNFS attack is restricted to one choice: $\operatorname{deg} h=k$. It leads to a smaller target finite field than in the composite cases. But a prime embedding degree eliminates some optimisation opportunities for the pairing computation.

This article also gives cost estimates and comparisons based on existing software. We show that the added confidence in the DL hardness can be obtained without sacrificing the pairing efficiency too much.

Organisation of the paper. In Section 2, we recall the Tate and ate pairings, in particular the notions of Miller loop and final exponentiation. We present in Section 3 our Cocks-Pinch variant to construct secure curves with an efficient pairing. Section 4 addresses the cost estimates for DL computation with the known variants of the NFS algorithm. In Section 5 we provide parameters of curves for 128 bits of security together with the analysis of the pairing cost. We compare the pairing efficiency to their challengers: BN, BLS12 and KSS16 curves, and embedding degree one curves from [16].

\subsection{Code repository}

Companion code is provided for several sections in this article, including code to reproduce experimental data. The code repository is publicly accessible at:

https://gitlab.inria.fr/smasson/cocks-pinch-variant.

\section{Background on pairings}

We present here the computation of two pairings used in practice, the Tate and ate pairings. Then we list refinements in the case of ate pairing on BN curves.

Let $E$ be an elliptic curve defined over $\mathbb{F}_{p}$. Let $\pi_{p}$ be the Frobenius endomorphism $(x, y) \mapsto\left(x^{p}, y^{p}\right)$. Its minimal polynomial is $X^{2}-t X+p$ and $t$ is 
called the trace. Let $r$ be a prime divisor of $\# E\left(\mathbb{F}_{p}\right)=p+1-t$. The $r$-torsion subgroup of $E$ is denoted $E[r]:=\left\{P \in E\left(\overline{\mathbb{F}_{p}}\right),[r] P=\mathcal{O}\right\}$ and has two subgroups of order $r$ (eigenspaces of $\pi_{p}$ in $E[r]$ ) that are useful for pairing applications: $\mathbb{G}_{1}=E[r] \cap \operatorname{ker}\left(\pi_{p}-[1]\right)$ and $\mathbb{G}_{2}=E[r] \cap \operatorname{ker}\left(\pi_{p}-[p]\right)$. The latter is defined over $\mathbb{F}_{p^{k}}$, where the embedding degree $k$ is the smallest integer $k \in \mathbb{N}^{*}$ such that $r$ divides $p^{k}-1$. A pairing-friendly curve is an elliptic curve that satisfies the following conditions: $p$ and $r$ are prime numbers, $t$ is relatively prime to $p$, and $k$ should be small. The discriminant $-D$ is the fundamental discriminant of the quadratic imaginary field defined by $X^{2}-t X+p$ (so that $t^{2}-4 p=-D y^{2}$ for an integer $y$ ). All constructions require that $|D|$ be small enough, so that the complex multiplication method is feasible (the record computation in [50] has $\left.|D| \sim 10^{16}\right)$. The $\rho$-value of $E$ is defined by $\rho(E)=\log (p) / \log (r)$. The "ideal" case is $\rho(E) \approx 1$ when $r=\# E\left(\mathbb{F}_{p}\right)$.

We recall the Tate and ate pairings definition, based on the same two steps: evaluating a function $f_{s, Q}$ at a point $P$, and then raising it to the power $\left(p^{k}-1\right) / r$. (Sometimes the pairing is said reduced to stress the final exponentiation). The function $f_{s, Q}$ has divisor $\operatorname{div}\left(f_{s, Q}\right)=s(Q)-([s] Q)-(s-1)(\mathcal{O})$ and satisfies for integers $i$ and $j$

$$
f_{i+j, Q}=f_{i, Q} f_{j, Q} \frac{\ell_{[i] Q,[j] Q}}{v_{[i+j] Q}}
$$

where $\ell_{[i] Q,[j] Q}$ and $v_{[i+j] Q}$ are the two lines needed to compute $[i+j] Q$ from $[i] Q$ and $[j] Q$ ( $\ell$ through the two points, $v$ the vertical). We compute $f_{s, Q}(P)$ with the Miller loop presented in Algorithm 1.

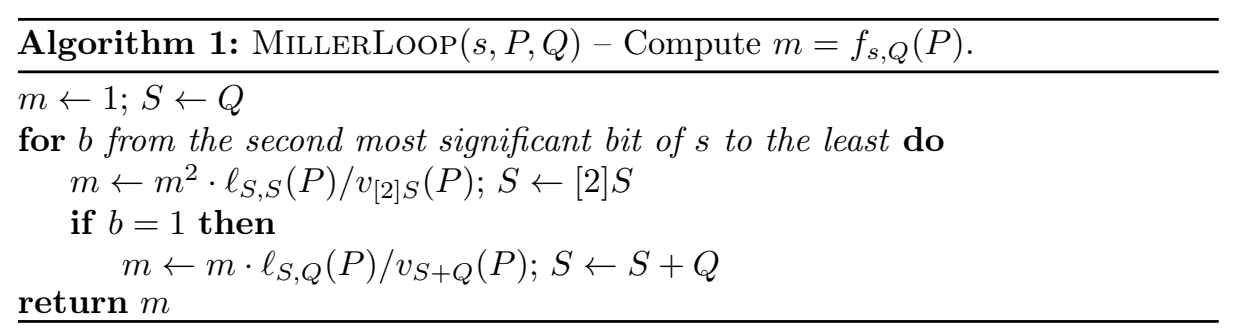

The Tate and ate pairings are defined by

$$
\operatorname{Tate}(P, Q):=f_{r, P}(Q)^{\left(p^{k}-1\right) / r}, \text { and } \operatorname{ate}(P, Q):=f_{t-1, Q}(P)^{\left(p^{k}-1\right) / r}
$$

where $P \in \mathbb{G}_{1} \subset E[r]\left(\mathbb{F}_{p}\right)$ and $Q \in \mathbb{G}_{2} \subset E[r]\left(\mathbb{F}_{p^{k}}\right)$. The values Tate $(P, Q)$, and ate $(P, Q)$ are in the "target" group $\mathbb{G}_{T}$ of $r$-th roots of unity in $\mathbb{F}_{p^{k}}$.

Before we analyse in Section 5 the cost of computing pairings, we briefly comment on the CM discriminant $-D$. When $D=3$ (resp. $D=4$ ), the curve has complex multiplication by $\mathbb{Q}(\sqrt{-3})$ (resp. $\mathbb{Q}(\sqrt{-1})$ ), so that a twist of degree 6 (resp. 4) exists. When $E$ has $d$-th order twists for some $d \mid k$, then $E[r]\left(\mathbb{F}_{p^{k}}\right)$ is isomorphic to $E^{\prime}[r]\left(\mathbb{F}_{p^{k / d}}\right)$ for some twist $E^{\prime}$. Dealing with the latter is easier. Therefore, composite extension degrees are often an invitation to choose $D=3$ or $D=4$. 


\section{Construction of secure curves with efficient ate pairing}

In this section, we look for curves that are not threatened by recent variants of NFS. We make the following observations.

- All families of curves in [26] compute $p$ as a polynomial evaluated at a chosen integer. This (often) enables the STNFS algorithm [33], so that the DL problem in $\mathbb{F}_{p^{k}}$ is easier than in other fields of same bit length.

- While composite extension degrees are appealing for fast pairing computation (see §2), they also offer additional parameterisation choices for the TNFS algorithm [36]. This also makes DL computations in $\mathbb{F}_{p^{k}}$ more efficient.

We wish to avoid special primes. Furthermore, as our range of interest $5 \leq k \leq 8$ contains the composite degrees $k=6$ and $k=8$, we acknowledge the need to choose the size of $p$ so as to compensate the TNFS attack.

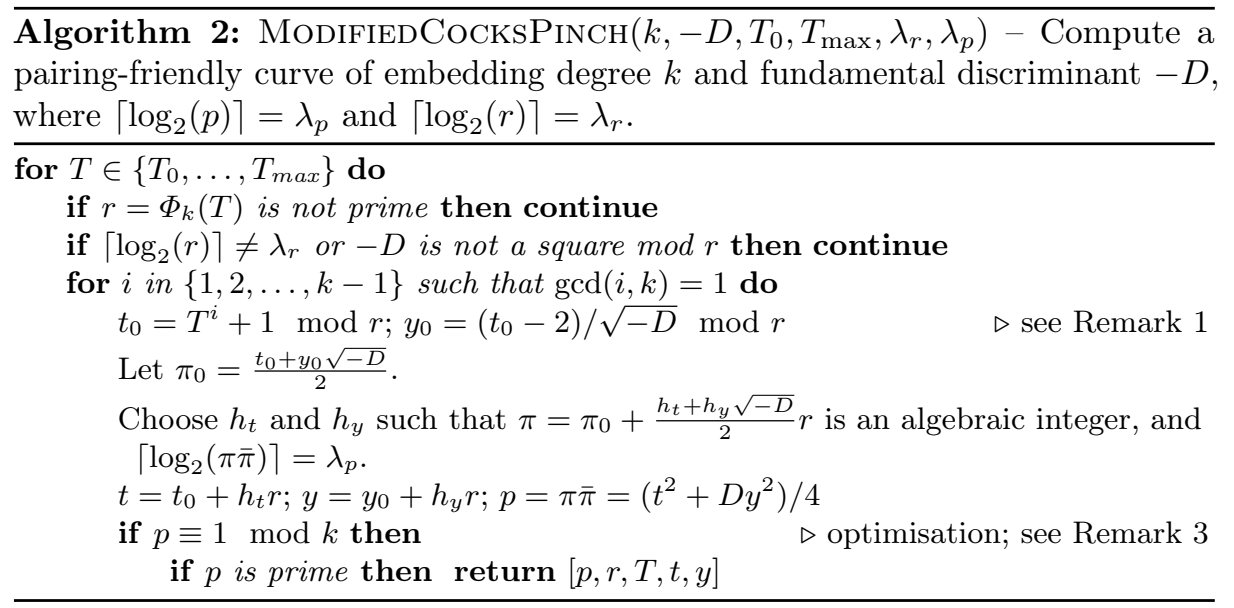

The special variants of NFS rely on a special form of $p$ : the prime integer should have a polynomial form $p=p(u)$ where $u$ is a seed and $p(x)$ is a polynomial. Roughly put, for the special variants to apply, $p(x)$ should have degree at least 3 and (most importantly) tiny coefficients. MNT curves of embedding degree $k=6$ are defined by a characteristic $p(x)=4 x^{2}+1$, a curve order $r(x)=4 x^{2}-2 x+1$ and a trace $t(x)=2 x+1$. One can check that $r(x) \mid \Phi_{6}(p(x))$ where $\Phi_{6}(x)=x^{2}-x+1$ is the 6 -th cyclotomic polynomial. Yet the prime $p$ of a MNT curve is not special because $p(x)$ has degree 2 only. BLS curves for $k=6$ have $p(x)=\left(x^{4}-3 x^{3}+4 x^{2}+1\right) / 3, r(x)=x^{2}-x+1$ and $t(x)=x+1$. The prime $p$ of a BLS- 6 curve is special because $p(x)$ has degree 4 and tiny coefficients (one can use $3 p(x)$ in the Special setting, and the largest coefficient is 4 ). We will obtain families of curves where $p(x)$ has a degree larger than 2 but has large coefficients so that the special setting (STNFS) does not perform better than a generic setting (TNFS). 
To avoid special primes, we revisit the Cocks-Pinch method, which constructs pairing-friendly curves with freely chosen embedding degree $k$ and discriminant $-D$. The classical Cocks-Pinch algorithm first fixes the prime $r$ and deduces a root of unity $\bmod r$ to compute $t$ and then $p$ satisfying the conditions of pairing-friendly curves. Instead, we first choose $T$ small, and then compute $r$ such that $T$ is a root of the $k$-th cyclotomic polynomial $\Phi_{k} \bmod r$. Then we observe that $f_{T, Q}(P)$ like $f_{t-1, Q}(P)$ gives a Miller loop of a bilinear pairing.

Our variant is given in Algorithm 2. The trace $\bar{t} \in \mathbb{Z} / r \mathbb{Z}$ can be any of $\bar{t}=T^{i}+1 \bmod r$ where $\operatorname{gcd}(i, k)=1$, and $\pm \bar{y}=(\bar{t}-2) / \pm \sqrt{-D} \bmod r$ as in the original method. We then lift $\bar{t}, \pm \bar{y}$ to $t_{0}, y_{0} \in \mathbb{Z}$. Since $\sqrt{-D}$ is only determined up to sign, we fix the sign indetermination problem as follows.

Remark 1 (Normalisation of $t_{0}$ and $y_{0}$ ). When lifting $\bar{t}, \pm \bar{y}$ from $\mathbb{Z} / r \mathbb{Z}$ to $\mathbb{Z}$, we choose $t_{0}$ as the signed representative of $\bar{t}$ with smallest absolute value, and $y_{0}$ as the smallest positive representative of $\pm \bar{y}$.

The choice of the cofactors $h_{t}$ and $h_{y}$ in Algorithm 2 must abide by certain rules so that the Weil number $\pi$ is an algebraic integer: if $-D \equiv 0 \bmod 4$, then $t_{0}+h_{t}$ must be even, and if $-D \equiv 1 \bmod 4$, then $t_{0}+y_{0}+h_{t}+h_{y}$ must be even. For $p$ to have the desired bit length, we notice that $h_{t}$ and $h_{y}$ must be chosen in an annuluslike region given by the equation $2^{\lambda_{p}+1} \leq\left(t_{0}+h_{t} r\right)^{2}+D\left(y_{0}+h_{y} r\right)^{2}<2^{\lambda_{p}+2}$.

The Miller algorithm in the ate pairing iterates on $t-1=T^{i}$, a $k$-th root mod $r$. Iterating on another root of unity also gives a pairing, as remarked by the following statement.

Theorem 2 ([52, §2.2]). Let $P \in \mathbb{G}_{1}=E[r] \cap \operatorname{ker}\left(\pi_{p}-[1]\right)$ and $Q \in \mathbb{G}_{2}=$ $E[r] \cap \operatorname{ker}\left(\pi_{p}-[p]\right)$. Let $T$ be a $k$-th root of unity mod $r$. Then, $f_{T, Q}(P)$ defines a bilinear pairing and

$$
\operatorname{Tate}(Q, P)^{L}=f_{T, Q}(P)^{c\left(p^{k}-1\right) / N}
$$

where $N=\operatorname{gcd}\left(T^{k}-1, p^{k}-1\right), T^{k}-1=L N$, and $c=\sum_{i=0}^{k-1} T^{k-1-i} p^{j i}$.

In particular, our $T$ in Algorithm 2 is convenient and defines an optimal ate pairing in the sense of [52, Definition 1]:

$$
\text { OptimalAte }(P, Q):=f_{T, Q}(P)^{\frac{p^{k}-1}{r}} \text {. }
$$

Does Algorithm 2 produce primes of a special form? In this paper, we will discuss in particular whether we hold to our promise that $p$, as issued by Algorithm 2, is not special. The prime $p$ has the form

$$
p=\frac{1}{4}\left(\left(t_{0}+h_{t} r\right)^{2}+D\left(y_{0}+h_{y} r\right)^{2}\right)
$$

where $t_{0}, y_{0}$ are centered representatives of $T^{i}+1 \bmod r$ and $\left(t_{0}-2\right) / \sqrt{-D} \bmod r$ resp., and both $r$ and $t_{0}$ are low-degree polynomials in $T$. 
If $T, h_{t}$, and $h_{y}$ are chosen by Algorithm 2 as random integers of the desired bit length, and that $D$ is arbitrary (then $y_{0}$ has no nice sparse polynomial expression in $T$ ), then the expression above is considered unlikely to yield any computational advantage to an attacker.

On the other hand, efficiency considerations may lead us to choose $D$ specially, so as to allow extra automorphisms on the curve, for example choose $-D$ as the discriminant of the $d$-th cyclotomic field $\mathbb{Q}\left(\zeta_{d}\right)$ for some $d \mid k$. Then $\sqrt{-D}$ typically has a low-degree polynomial expression in $T$. If $T, h_{t}$, and $h_{y}$ are then chosen with low Hamming weight, the answer is less clear. In comparison to other pairing-based constructions however (see Table 2), we have here a multivariate expression for $p$ (it depends on $T, h_{t}$, and $h_{y}$ ). First there exists no specialpurpose NFS construction that adapts well to a bivariate polynomial. Secondly for fixed $h_{t}$ and $h_{y}$, one can obtain a univariate polynomial in $T$. This setting will provide a notable advantage to NFS only if $h_{t}$ and $h_{y}$ are tiny enough to produce a sparse polynomial $p_{h_{t}, h_{y}}(T)$. As an illustration, here is the multivariate expression of $4 p$ in the case $k=8, D=4$, and $i=1$.

$$
\begin{aligned}
4 p= & \left(h_{t}^{2}+4 h_{y}^{2}\right) T^{8}+4 h_{y} T^{7}-\left(4 h_{y}-1\right) T^{6}+2\left(h_{t}-1\right) T^{5} \\
& +\left(2 h_{t}^{2}+8 h_{y}^{2}+2 h_{t}+1\right) T^{4}+4 h_{y} T^{3}-\left(4 h_{y}-1\right) T^{2}+2\left(h_{t}+1\right) T \\
& +\left(h_{t}^{2}+4 h_{y}^{2}+2 h_{t}+1\right) .
\end{aligned}
$$

If $h_{t}$ and $h_{y}$ are small, one can get a univariate expression for $p$ and exploit the $\mathrm{S}(\mathrm{T})$ NFS variant. In [24, Remark 3, Table 3], one finds a family (denoted FK-8 in the following to stand for Fotiadis-Konstantinou) with $D=4, h_{t}=1, h_{y}=0$ and $p(x)=\left(x^{8}+x^{6}+5 x^{4}+x^{2}+4 x+4\right) / 4, r(x)=x^{4}+1, t(x)=x+1+r(x)=x^{4}+x+2$, $y(x)=\left(x^{3}-x^{2}\right) / 2\left(\right.$ where $\left.p(x)=\left(t(x)^{2}+4 y(x)^{2}\right) / 4\right)$. In our case, for $r$ of 256 bits and $p$ of 544 bits, we have $h_{y}$ of 16 bits, hence $p_{h_{t}, h_{y}}(T)$ has coefficients of more than 32 bits.

We design in Section 4 an estimation of the cost needed to compute a discrete logarithm in $\mathbb{F}_{p^{k}}$ with STNFS and TNFS and compare the costs obtained for MNT-6, BLS-6 and Cocks-Pinch-6 curves, and FK-8, Cocks-Pinch- 8 curves and a third $k=8$ family of curves (Tanaka-Nakamula curves). We show in $\S 4.2$ as well as in $\S \mathrm{C}$ that using very small $h_{t}$ and $h_{y}$, the NFS variants can exploit a univariate polynomial expression and reduce the security of the DL over the finite field $\mathbb{F}_{p^{k}}$. But if these parameters are large enough, the advantage vanishes.

\section{DL cost estimate and size requirements}

In this section, we would like to determine, for each embedding degree $k \in$ $\{5,6,7,8\}$, the appropriate bit length of $p$ so that the pairings on the curves constructed in $\S 3$ match the 128-bit security level. This leads us to assess the hardness of the DL computation in the subgroup of (256-bit) prime order $r$ of $\mathbb{F}_{p^{k}}$. In terms of notations, we naturally search for different values of $p$ for each $k$, so that $p$ depends on $k$. For brevity however, we prefer to keep the notation $p$ rather than use $p_{k}$. 
We also do the same analysis for other curve families. We strive to take into account in our analysis the different known NFS variants. This complements the study in [7].

\subsection{Strategy for estimating NFS cost}

Estimating the computational cost of the number field sieve is a delicate task because of the inherent complexity of NFS, its numerous parameters and its variants, and moreover because of the very different nature of the different steps of the algorithm. This is a vastly different situation from, say, the assessment of the DLP hardness for elliptic curves, where the lack of any algorithm more advanced than $O(\sqrt{r})$ algorithms makes estimations comparatively easy.

The two main steps of NFS are relation collection and linear algebra. We tried to estimate both, in terms of "elementary operations" that combine a memory access (to sieve array elements, to vector coefficients) as well as arithmetic operations. Our simulation methodology is not dissimilar to the one used in [42,7]: starting from an NFS or TNFS setup, we estimate the norms involved in the relation collection step, and the associated smoothness probability. Further detail is given in Appendix B.2. We summarise here the variations that we introduce:

- In the NFS context, coprime $(a, b)$ pairs that form the primary source of candidates for smoothness are counted as the total number of pairs in the sieve area times the factor $\frac{6}{\pi^{2}}=\frac{1}{\zeta(2)}$. In the TNFS context, the analogue of this scaling is given by $\frac{1}{\zeta_{K}(2)}$ where $\zeta_{K}$ is the Dedekind zeta function of the base number field [30].

- We compute the smoothness probabilities of the two norms that are deduced from $(a, b)$ pairs as the average of the smoothness probabilities of norms over $10^{6}$ samples, instead of the smoothness probabilities of the average norm over 25600 samples.

- Estimating the matrix size that results from a given set of parameter choices requires to estimate the reduction factor of the so-called filtering step. As we show in Appendix B.2, recent large computations chose parameters very differently, which led to vastly different reduction factors. Based on a rationale for parameter choice that is in accordance with previous computation with the cado-nf s software, we estimate the filtering step as providing a (conservative) constant reduction factor of 9 . This is very different from the reasoning in [7] and we justify this choice in Appendix B.2.

\subsection{Evolution of DL cost as $h_{t}, h_{y}$ increase for $k=6,8$}

Embedding Degree 6: MNT-6, BLS-6 and Cocks-Pinch-6 Curves. We wish to compare the effectiveness of various NFS strategies, for our Cocks-Pinch-6 curves having $D=3$, as well as other curves. Targeting the 128-bit security level, we fix the size of $r$ in Cocks-Pinch-6 curves to be 256 bits, hence $T$ is 64 bits long. We set $D=3$, and let $p$ vary from 512 to 800 bits thanks to $h_{y}$ of increasing size from 1 to 145 bits. For comparison, we generate MNT-6 
parameters with Ben Lynn's pbc library where $p$ runs from 126 to 2047 bits, and BLS-6 parameters where $p$ runs from 126 to 2046 bits ( $r$ is 64 to 1024 bit long). Then we run the STNFS and TNFS DL cost estimate for each $p$. The results are presented in Figure 3, where the degree of $h$ is implicitly chosen so as to minimise the estimated cost of (S)TNFS, and the notation $L_{N}^{0}$ stresses the fact that the asymptotic complexity is used with $o(1)$ set to 0 .

MNT-6 and Cocks-Pinch- 6 curves of $p$ of 672 bits ( $p^{k}$ of 4032 bits) provide 128 bits of security, while a BLS- 6 curve of that size only provides 116 bits of security, a 832-bit $p$ is needed for a BLS-6 curve to provide 128 bits of security. The polynomials for TNFS are given in Table 15. All parameters can be obtained in the code repository mentioned in $§ 1.1$.

$\longrightarrow$ Simulation in $\mathbb{F}_{p^{6}}$, Cocks-Pinch-6, TNFS-conj deg $h=2$

$\diamond$ Simulation in $\mathbb{F}_{p^{6}}$, Cocks-Pinch-6, STNFS-JP $\operatorname{deg} h=3$

- Simulation in $\mathbb{F}_{p^{6}}$, MNT6, TNFS-conj $\operatorname{deg} h=2$

$\multimap$ Simulation in $\mathbb{F}_{p^{6}}$, BLS-6, STNFS-JP $\operatorname{deg} h \in\{6,3,2\}$

$-L_{N}^{0}(1 / 3,1.923) / 2^{8.2}$ (NFS, theoretical, re-scaled s.t. DL-768 $\leftrightarrow 2^{68.32}$ )

- - - $L_{p^{n}}^{0}(1 / 3,1.526) / 2^{4.5}$ (SNFS, theoretical re-scaled SDL-1024 $\leftrightarrow 2^{64.4}$ )

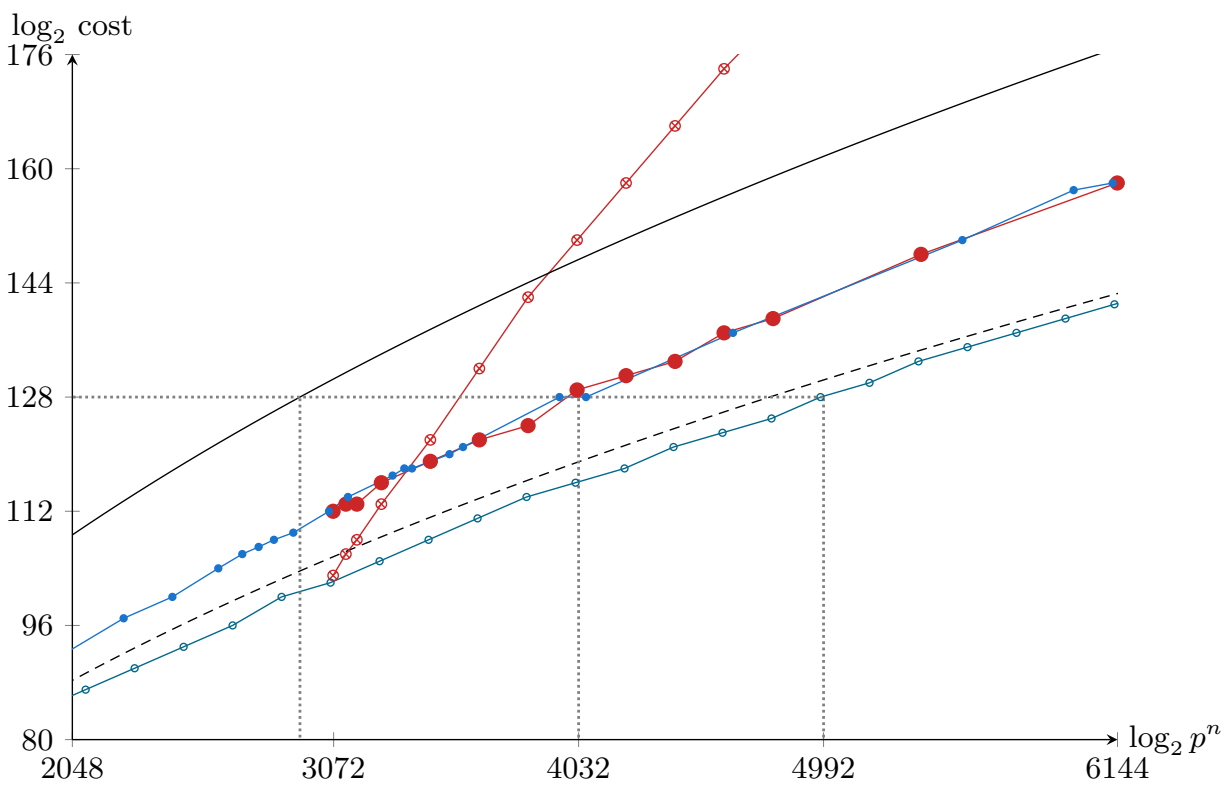

Fig. 3: TNFS vs STNFS simulations for curves with $k=6$.

Embedding Degree 8: Tanaka-Nakamula, Fotiadis-Konstantinou and Cocks-Pinch-8 Curves. We do the same for $k=8$. We generate Cocks-Pinch- 
8 parameters with $D=4, r$ of fixed length 256 bits, and $h_{y}$ growing from 1 to 128 bits, so that $p$ grows from 512 to 768 bits. We compare to Tanaka-Nakamula curves of $k=8$ (TN-8) [51] where $p(x)$ has degree 6, and Fotiadis-Konstantinou curves of $k=8$ and $D=4(\mathrm{FK}-8)$ [24, Table 3] where $p(x)$ has degree 8 . We obtain Figure 4. Cocks-Pinch- 8 curves of $r$ of 256 bits and $p$ of 544 bits $\left(h_{t}, h_{y}\right.$ of 16 bits) provide 128 bits of security. TN- 8 curves of $p$ of 544 bits have $r$ of 362 bits and offer 125 bits of security. FK-8 curves of $p$ of 544 bits have $r$ of 273 bits and provide 116 bits of security. To obtain 128 bits of security, TN-8 curves should have $p$ of 576 bits (then $r$ is 383 bits long) and FK-8 curves should have $p$ of 672 bits (then $r$ is 337 bits long).

\footnotetext{
$\longrightarrow$ Simulation in $\mathbb{F}_{p^{8}}$, Cocks-Pinch, TNFS-conj deg $h=2$

$\rightarrow$ Simulation in $\mathbb{F}_{p^{8}}$, Cocks-Pinch, STNFS-JP $\operatorname{deg} h=8, r$ of 256 bits

- Simulation in $\mathbb{F}_{p^{8}}$, Tanaka-Nakamula, STNFS-JP $\operatorname{deg} p=6, \operatorname{deg} h=4$

$\longrightarrow$ Simulation in $\mathbb{F}_{p^{8}}$, Fotiadis-Konstantinou, STNFS-JP $\operatorname{deg} p=8, \operatorname{deg} h=8$

$-L_{p}^{0}(1 / 3,1.923) / 2^{8.2}$ (NFS, theoretical, re-scaled s.t. DL-768 $\left.\leftrightarrow 2^{68.32}\right)$

- - - $L_{p^{n}}^{0}(1 / 3,1.526) / 2^{4.5}$ (SNFS, theoretical re-scaled SDL-1024 $\leftrightarrow 2^{64.4}$ )
}

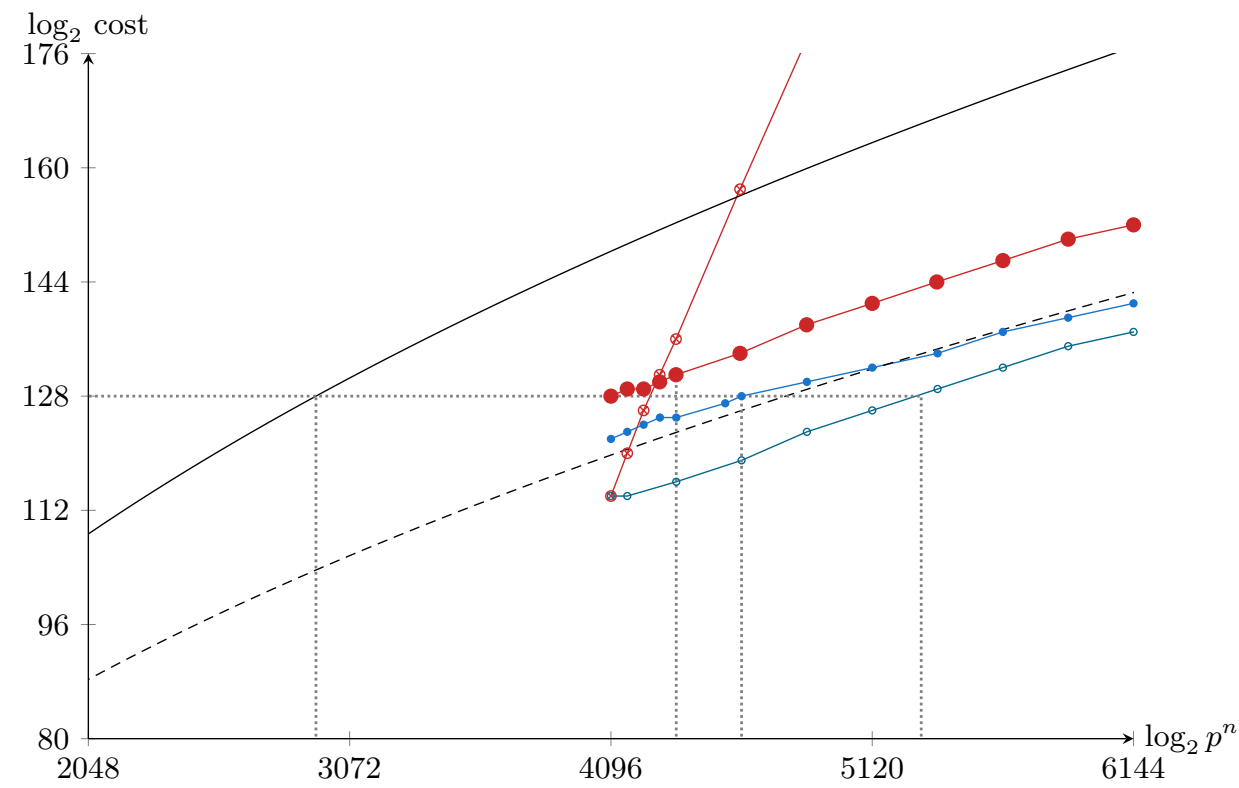

Fig. 4: TNFS vs STNFS simulation for curves with $k=8$.

Evolution of DL cost as $\log _{2} p$ changes for $k=6,8$. It follows from Figures 3 and 4 that the special form of $p$ for our curves does offer an advantage compared to the generic Conjugation-TNFS algorithm only for very small sizes 
of $p$, between 512 and 560 bits for $k=6, D=3$ (this corresponds to $\left|h_{t}\right|$ and $\left|h_{y}\right|$ of at most 20 bits), and between 512 and 536 bits for $k=8, D=4\left(\left|h_{t}\right|\right.$ and $\left|h_{y}\right|$ of at most 12 bits). For larger parameters, the generic TNFS algorithm is faster, which supports our claim that the primes that we use are not special.

\subsection{Cost estimates at the 128-bit security level}

Our simulation results are given in Table 5, which covers both the method in $\S 3$ as well as competing curves. In each case, only the most efficient NFS variant is retained. For reference, we also include a cost estimate, obtained with the same method, for 3072-bit prime fields. This fits reasonably well with the commonly accepted idea that this size matches 128 -bit security (see e.g. [44, §5.6]).

In addition to the parameters for BLS12 and BN curves that were proposed in [7], we find it necessary to also consider curves in these families for a 446-bit prime field. As it turns out, this gives only a marginally different cost estimate for the attack, and the fact that $p$ fits in seven 64-bit machine words is a clear implementation advantage. For BN-446 the seed $u=2^{110}+2^{36}+1$ is taken from [45] and our seed for BLS12-446 is $u=-\left(2^{74}+2^{73}+2^{63}+2^{57}+2^{50}+2^{17}+1\right)$ so that the curve is subgroup-, twist-, and subgroup-twist-secure.

\begin{tabular}{l|l|ccccccc} 
Original family & curve or field & $k$ & $\log _{2} p^{k}$ & $\log _{2} r$ & $\mathrm{~d} h$ & $\mathrm{~d} f, \mathrm{~d} g$ & $\mathrm{poly}$ & cost \\
\hline prime fields & Oakley $p$ & 1 & 3072 & 3071 & - & $(5,4)$ & $\mathrm{JL}$ & $2^{127}$ \\
& prime field & 1 & 3200 & 256 & - & $(5,4)$ & $\mathrm{JL}$ & $2^{128}$ \\
\hline$[16]$ & & 1 & 3072 & 256 & - & $(5,4)$ & $\mathrm{JL}$ & $2^{128}$ \\
\hline This work & $k 5, p 663$ & 5 & 3318 & 256 & 5 & $(6,5)$ & $\mathrm{JL}$ & $2^{128}$ \\
& $k 6, p 672, D=3$ & 6 & 4028 & 256 & 2 & $(6,3)$ & $\mathrm{Conj}$ & $2^{128}$ \\
& $k 7, p 512$ & 7 & 3584 & 256 & 7 & $(6,5)$ & $\mathrm{JL}$ & $2^{132}$ \\
& $k 8, p 544, D=4$ & 8 & 4349 & 256 & 2 & $(8,4)$ & $\mathrm{Conj}$ & $2^{131}$ \\
\hline$[26$, Ex. 6.8] & BN-446 & 12 & 5343 & 446 & 6 & $(8,2)$ & $\mathrm{JP}$ & $2^{132}$ \\
& BN-462 & 12 & 5534 & 462 & 6 & $(8,2)$ & $\mathrm{JP}$ & $2^{135}$ \\
\hline$[26, \S 6.1]$ & BLS12-381 & 12 & 4572 & 255 & 6 & $(12,2)$ & $\mathrm{JP}$ & $2^{126}$ \\
& BLS12-446 & 12 & 5352 & 299 & 6 & $(12,2)$ & $\mathrm{JP}$ & $2^{132}$ \\
& BLS12-461 & 12 & 5525 & 309 & 6 & $(12,2)$ & $\mathrm{JP}$ & $2^{134}$ \\
\hline$[26$, Ex. 6.11] & KSS16-330 & 16 & 5280 & 257 & 16 & $(10,1)$ & $\mathrm{JP}$ & $2^{141}$ \\
& KSS16-339 & 16 & 5411 & 263 & 16 & $(10,1)$ & $\mathrm{JP}$ & $2^{141}$
\end{tabular}

Table 5: Comparison of DL cost estimates. Polynomial selection methods are abbreviated as Conj for the conjugation method [8], JL for Joux-Lercier [32], JP for the Joux-Pierrot STNFS variant [33] (when it improves on JL).

\section{Pairing cost}

We now count the number of operations over $\mathbb{F}_{p}$ to compute the optimal ate pairing with Algorithms 3, 4 and 5 . We denote by $\mathbf{m}_{k}, \mathbf{s}_{k}, \mathbf{i}_{k}$ and $\mathbf{f}_{k}$ the costs 
of multiplication, squaring, inversion, and $p$-th power Frobenius in $\mathbb{F}_{p^{k}}$, and by $\mathbf{m}=\mathbf{m}_{1}$ the multiplication in $\mathbb{F}_{p}$. We neglect additions and multiplications by small constants. In order to provide a common comparison base, we give estimated costs for $\mathbf{m}_{k}$ and $\mathbf{s}_{k}$ using Karatsuba-like formulas [22,43,19]. Inversions are computed using the expression below, which relies on efficient Frobenius powers:

$$
a^{-1}=\left(\operatorname{Norm}_{\mathbb{F}_{q}^{k} / \mathbb{F}_{q}}(a)\right)^{-1} \times a^{q} \times \cdots \times a^{q^{k-1}} .
$$

Remark 3 (Frobenius cost). For the latter to perform well, it is very useful to have $p \equiv 1 \bmod k$ (as we did in Algorithm 2), and define $\mathbb{F}_{p^{k}}$ as $\mathbb{F}_{p^{k}}=\mathbb{F}_{p}[x] /\left(x^{k}-\alpha\right)$ : let $w=\sum_{i=0}^{k-1} \omega_{i} x^{i} \in \mathbb{F}_{p^{k}}=\mathbb{F}_{p}[x] /\left(x^{k}-\alpha\right)$. Then, $w^{p^{j}}=\omega_{0}+\sum_{i=1}^{k-1} \omega_{i} x^{i p^{j}}$. The terms $x^{i p^{j}}$ do not depend on $w$ and are precomputed. By Euclidean division by $k, x^{i p^{j}}=x^{u_{j} k+i}=\alpha^{u_{j}} x^{i}$. Therefore we have at most $\mathbf{f}_{k}=(k-1) \mathbf{m}$ for any $p^{j}$-th power Frobenius. Note that for $k$ even, we have $x^{k / 2 \cdot\left(p^{j}-1\right)}=\alpha^{\left(p^{j}-1\right) / 2}= \pm 1$ so that $x^{k / 2 \cdot p^{j}}= \pm x^{k / 2}$, whence one multiplication can be saved.

Consequences of the above are given in [48], notably $\mathbf{i}_{2}=2 \mathbf{m}+2 \mathbf{s}_{1}+\mathbf{i}_{1}$ and $\mathbf{i}_{3}=9 \mathbf{m}+3 \mathbf{s}_{1}+\mathbf{i}_{1}$, neglecting additions. Recursive application yields $\mathbf{i}_{k}$ for $k=2,3,4,6,8,12,16$. For $k=5$, we compute $t:=a^{q} \times \cdots \times a^{q^{4}}=\left(\left(a^{q}\right)^{1+q}\right)^{1+q^{2}}$ and then use the fact that the norm of $a$ is in $\mathbb{F}_{p}$ to get $\operatorname{Norm}_{\mathbb{F}_{p^{5}} / \mathbb{F}_{p}}(a)=a \times t=$ $a_{0} t_{0}+\alpha \sum_{j=1}^{k-1} a_{i} t_{k-i}$. We finally obtain that $\mathbf{i}_{5}=3 \mathbf{f}_{5}+2 \mathbf{m}_{k}+\mathbf{i}_{1}+10 \mathbf{m}$, and $\mathbf{i}_{7}$ is obtained in a similar way. Table 6 summarises these estimated costs, and includes specialised costs for cyclotomic squares (see $[29, \S 3.1]$ ). We compared Table 6 with timings of the RELIC library [4] for primes $p$ of 6 to 8 machine words and $k=2,6,12$ on an Intel Core i5-4570 CPU, 3.20GHz. The accordance is satisfactory (within 10\%), to the point that we use Table 6 as a base. Additionally, we also measured the relative costs of $\mathbf{i}_{1}, \mathbf{s}_{1}$, and $\mathbf{m}$ on the same platform ${ }^{3}$, leading to $\mathbf{i}_{1} \approx 25 \mathbf{m}$ and $\mathbf{s}_{1} \approx \mathbf{m}$.

\begin{tabular}{|c|c|c|c|c|c|c|c|c|c|}
\hline$k$ & 1 & 2 & 3 & 5 & 6 & 7 & 8 & 12 & 16 \\
\hline $\mathbf{m}_{k}$ & $\mathbf{m}$ & $3 \mathbf{m}$ & $6 \mathbf{m}$ & $13 \mathbf{m}$ & $18 \mathbf{m}$ & $22 \mathbf{m}$ & $27 \mathbf{m}$ & $54 \mathbf{m}$ & $81 \mathbf{m}$ \\
$\mathbf{s}_{k}$ & $\mathbf{m}$ & $2 \mathbf{m}$ & $5 \mathbf{m}$ & $13 \mathbf{m}$ & $12 \mathbf{m}$ & $22 \mathbf{m}$ & $18 \mathbf{m}$ & $36 \mathbf{m}$ & $54 \mathbf{m}$ \\
$\mathbf{f}_{k}$ & 0 & 0 & $2 \mathbf{m}$ & $4 \mathbf{m}$ & $4 \mathbf{m}$ & $6 \mathbf{m}$ & $6 \mathbf{m}$ & $10 \mathbf{m}$ & $14 \mathbf{m}$ \\
$\mathbf{s}_{k}^{\text {cyclo }}$ & & & & & $6 \mathbf{m}$ & & $12 \mathbf{m}$ & $18 \mathbf{m}$ & $36 \mathbf{m}$ \\
$\mathbf{i}_{k}-\mathbf{i}_{1}$ & 0 & $4 \mathbf{m}$ & $12 \mathbf{m}$ & $48 \mathbf{m}$ & $34 \mathbf{m}$ & $104 \mathbf{m}$ & $44 \mathbf{m}$ & $94 \mathbf{m}$ & $134 \mathbf{m}$ \\
$\mathbf{i}_{k}$, with $\mathbf{i}_{1}=25 \mathbf{m}$ & $25 \mathbf{m}$ & $29 \mathbf{m}$ & $37 \mathbf{m}$ & $73 \mathbf{m}$ & $59 \mathbf{m}$ & $129 \mathbf{m}$ & $69 \mathbf{m}$ & $119 \mathbf{m}$ & $159 \mathbf{m}$ \\
\hline
\end{tabular}

Table 6: Relative cost of $\mathbf{m}_{k}, \mathbf{s}_{k}$ and $\mathbf{i}_{k}$ for our finite field extensions.

\footnotetext{
${ }^{3}$ The approximation $\mathbf{i}_{1} \approx 25 \mathrm{~m}$ in Table 6 is clearly implementation-dependent. Since it has negligible bearing on the final cost anyway, we stick to that coarse estimate.
} 


\section{$5.1 \quad$ Miller loop}

The Miller loop evaluates functions defined over $\mathbb{F}_{p^{k}}$, at a point of $E\left(\mathbb{F}_{p}\right)$. Algorithm 3 is essentially a repetition of Algorithm 1 with a few modifications related to practice: it is desirable to separate numerators and denominators so as to compute only one inversion at the end. Furthermore, the argument $T$ may conveniently be handled in binary non-adjacent form $T=\sum_{i=0}^{n} b_{i} 2^{i}=\left(b_{n} b_{n-1} \ldots b_{2} b_{1} b_{0}\right)_{2 \text {-NAF }}$ with $b_{i} \in\{-1,0,1\}$. We use the notation $\mathrm{HW}_{2 \text {-NAF }}$ for the number of non-zero $b_{i}$.

Algorithm 3 uses three helper functions that are detailed as Algorithms 4 and 5. The input point $S$ as well as the output point $\mathbf{S}$ in these algorithms are in Jacobian coordinates [20]: the quadruple $\left(X, Y, Z, Z^{2}\right)$ represents the affine point $\left(X / Z^{2}, Y / Z^{3}\right)$. This saves inversions and multiplications.

In Table 7 , we give the cost of the line computations for $5 \leq k \leq 8$. As it turns out, the embedding degree $k$ affects the pairing cost in multiple ways. As mentioned in $\S 2$ (see also $[21,3]$ ), twists allow efficient computations for $6 \mid k$ (resp. $4 \mid k$ ) if we set $D=3$ (resp. $D=4$ ). During Algorithm 3, the factors in proper subfields of $\mathbb{F}_{p^{k}}$ are neutralised during the final exponentiation, and hence are not computed. In particular, $\lambda_{d}$ and $m_{d}$ are omitted from the Miller loop computation for these curves. Algorithms 4 and 5 are also simplified ${ }^{4}$. Table 7 takes adaptations of these optimisations into account when twists are available ${ }^{5}$, while the data for $k=5$ and $k=7$ comes straight from Algorithms 3, 4 and 5. We denote by $\mathbf{m c}_{k}$ the cost of multiplying a constant $c \in \mathbb{F}_{p}$ by an element of $\mathbb{F}_{p^{k}}$. A consequence of Table 7 is that the Jacobi quartic, Hessian, and Edwards models induce comparatively expensive pairing computations, and the Weierstrass model is preferred in practice.

The final cost of Algorithm 3 is given by the following formula, where the notation $\mathbf{c}_{X}$ denotes the cost of step $X$, or algorithm $X$.

$$
\begin{aligned}
\mathbf{c}_{\text {MillerLoop }}= & \left(\log _{2}(T)-1\right)\left(\mathbf{c}_{\text {DoubleLine }}+\mathbf{c}_{\text {VerticalLine }}\right) \\
& +\left(\log _{2}(T)-2\right) \mathbf{c}_{\text {Update1 }} \\
& +\left(\operatorname{HW}_{2-\mathrm{NAF}}(T)-1\right)\left(\mathbf{c}_{\text {AddLine }}+\mathbf{c}_{\text {VerticalLine }}+\mathbf{c}_{\text {Update } 2}\right) \\
& +(\text { only if } k \in\{5,7\}) \mathbf{i}_{k} .
\end{aligned}
$$

\footnotetext{
${ }^{4}$ In particular, the line computations involve some sparse products, e.g. $\left(\sum a_{i} x^{i}\right) \times$ $\left(\sum b_{i} x^{i}\right)$ in $\mathbb{F}_{p^{8}}$ over $\mathbb{F}_{p^{2}}$ with $a_{1}=0$ (see [21]), which costs $8 \mathbf{m}_{2}$ by Karatsuba. Note that [54] claims $7 \mathbf{m}_{2}$ but with no explicit formula. We were not able to match this. The work [35, §3.3] obtained $7 \mathbf{m}_{2}$ in favorable cases at a cost of extra precomputations.

${ }^{5}$ The Edwards model is not available for a quartic or sextic twist because there is no 4-torsion point on these twists, only the quadratic twist can be in Edwards form [41]. The Jacobi quartic model is not available for a cubic or sextic twist because there is no 2-torsion point on the twist. The Hessian model is compatible with cubic twists but not sextic twists.
} 


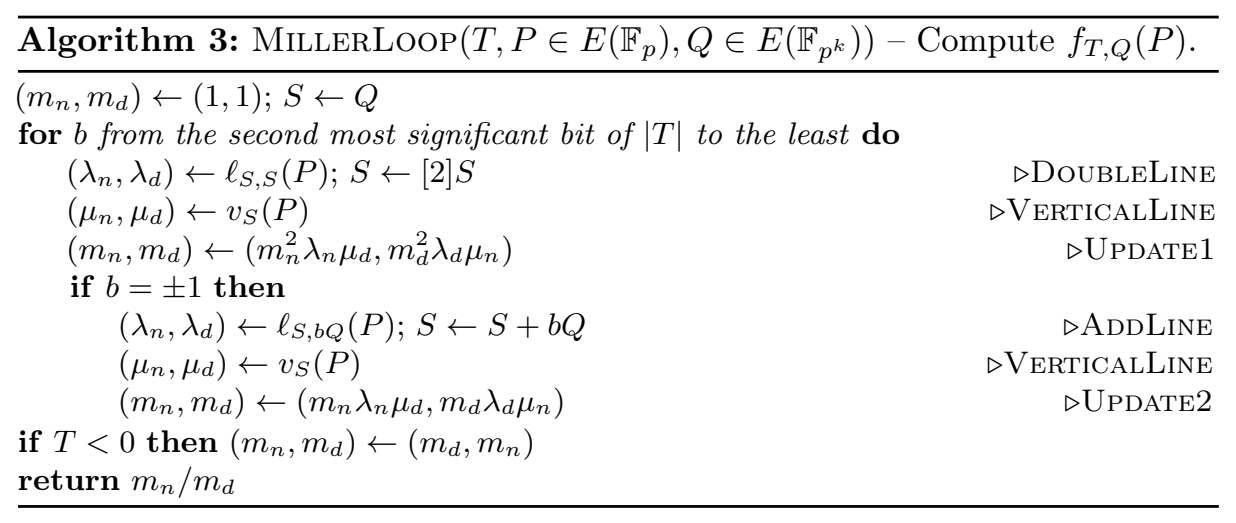

Algorithm 4: $\operatorname{Add} \operatorname{dine}(S, Q, P)$ and $\operatorname{Doubleline}(S, P)-$ Given $S, Q \in$ $E\left(\mathbb{F}_{p^{k}}\right)$, compute $S+Q$ (resp. $2 S$ ) and the evaluation of the line $(S Q)$ (resp. the tangent at $S)$ at $P \in E\left(\mathbb{F}_{p}\right)$.

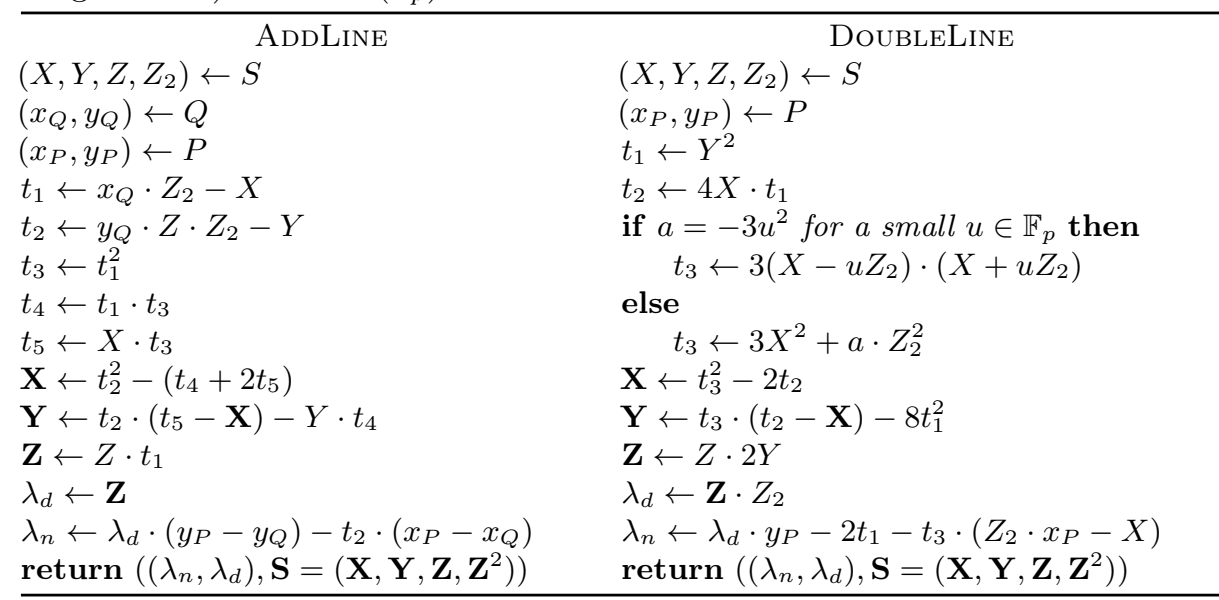




\begin{tabular}{|c|c|c|c|c|c|}
\hline$k$ & $-D$ & $\begin{array}{c}\text { ADDLine } \\
\text { and DoubleLine }\end{array}$ & \begin{tabular}{|c|} 
VERTICAL \\
LINE
\end{tabular} & $\begin{array}{c}\text { UPDATE1 } \\
\text { and UPDATE2 }\end{array}$ & ref \\
\hline \multicolumn{6}{|c|}{ Weierstrass model } \\
\hline 5 & any & $\begin{array}{c}10 \mathbf{m}_{5}+3 \mathbf{s}_{5} \\
6 \mathbf{m}_{5}+4 \mathbf{s}_{5}+10 \mathbf{m}\end{array}$ & $5 \mathbf{m}$ & $\begin{array}{l}4 \mathbf{m}_{5}+2 \mathbf{s}_{5} \\
4 \mathbf{m}_{5}\end{array}$ & Alg. 4,5 \\
\hline 7 & any & $\begin{array}{c}10 \mathbf{m}_{7}+3 \mathbf{s}_{7} \\
6 \mathbf{m}_{7}+4 \mathbf{s}_{7}+14 \mathbf{m}\end{array}$ & $7 \mathbf{m}$ & $\begin{array}{l}4 \mathbf{m}_{7}+2 \mathbf{s}_{7} \\
4 \mathbf{m}_{7}\end{array}$ & Alg. 4,5 \\
\hline $6|k|$ & -3 & $\begin{array}{c}10 \mathbf{m}_{k / 6}+2 \mathbf{s}_{k / 6}+(k / 3) \mathbf{m} \\
2 \mathbf{m}_{k / 6}+7 \mathbf{s}_{k / 6}+(k / 3) \mathbf{m}\end{array}$ & 0 & $\begin{array}{c}\mathbf{s}_{k}+13 \mathbf{m}_{k / 6} \\
13 \mathbf{m}_{k / 6} \\
\end{array}$ & {$[21, \S 5]$} \\
\hline $6|k|$ & -3 & $\begin{array}{c}11 \mathbf{m}_{k / 6}+2 \mathbf{s}_{k / 6}+(k / 3) \mathbf{m} \\
3 \mathbf{m}_{k / 6}+6 \mathbf{s}_{k / 6}+(k / 3) \mathbf{m}\end{array}$ & 0 & $\begin{array}{c}\mathbf{s}_{k}+13 \mathbf{m}_{k / 6} \\
13 \mathbf{m}_{k / 6}\end{array}$ & {$[5, \S 4,6]$} \\
\hline $4|k|$ & -4 & $\begin{array}{l}9 \mathbf{m}_{k / 4}+5 \mathbf{s}_{k / 4}+(k / 2) \mathbf{m} \\
2 \mathbf{m}_{k / 4}+8 \mathbf{s}_{k / 4}+(k / 2) \mathbf{m}\end{array}$ & 0 & $\begin{array}{c}\mathbf{s}_{k}+8 \mathbf{m}_{k / 4} \\
8 \mathbf{m}_{k / 4}\end{array}$ & {$[21, \S 4]$} \\
\hline \multicolumn{6}{|c|}{ Jacobi quartic model (not for cubic or sextic twist) } \\
\hline $4|k|$ & -4 & $\begin{array}{l}12 \mathbf{m}_{k / 4}+7 \mathbf{s}_{k / 4}+1 \mathbf{m c}_{k / 4}+(k / 2) \mathbf{m} \\
3 \mathbf{m}_{k / 4}+7 \mathbf{s}_{k / 4}+1 \mathbf{m} \mathbf{c}_{k / 4}+(k / 2) \mathbf{m}\end{array}$ & 0 & $\begin{array}{c}\mathbf{s}_{k}+8 \mathbf{m}_{k / 4} \\
8 \mathbf{m}_{k / 4}\end{array}$ & [23] \\
\hline $2|k|$ & any & $\begin{array}{c}16 \mathbf{m}_{k / 2}+1 \mathbf{s}_{k / 2}+4 \mathbf{m c}_{k / 2}+k \mathbf{m} \\
4 \mathbf{m}_{k / 2}+8 \mathbf{s}_{k / 2}+1 \mathbf{m c}_{k / 2}+k \mathbf{m}\end{array}$ & 0 & $\begin{array}{c}\mathbf{s}_{k}+\mathbf{m}_{k} \\
\mathbf{m}_{k}\end{array}$ & {$[25, \S 3.2]$} \\
\hline \multicolumn{6}{|c|}{ Hessian model (not for quartic twist) } \\
\hline $6|k|$ & -3 & $\begin{array}{c}7 \mathbf{m}_{k / 3}+4 \mathbf{m}_{k / 6}+(2 k / 3) \mathbf{m} \\
2 \mathbf{m}_{k / 6}+\mathbf{s}_{k / 6}+4 \mathbf{m}_{k / 3}+2 \mathbf{s}_{k / 3}+(k / 2) \mathbf{m}\end{array}$ & 0 & $\begin{array}{c}\mathbf{s}_{k}+13 \mathbf{m}_{k / 6} \\
2 \mathbf{m}_{k / 3}\end{array}$ & [18] \\
\hline \multicolumn{6}{|c|}{ Edwards model (not for quartic, cubic or sextic twist) } \\
\hline $2|k|$ & any & $\begin{array}{c}12 \mathbf{m}_{k / 2}+\mathbf{m c}_{k / 2}+k \mathbf{m} \\
4 \mathbf{m}_{k / 2}+7 \mathbf{s}_{k / 2}+2 \mathbf{m} \mathbf{c}_{k / 2}+k \mathbf{m}\end{array}$ & 0 & $\begin{array}{c}\mathbf{s}_{k}+\mathbf{m}_{k} \\
\mathbf{m}_{k}\end{array}$ & {$[41,25]$} \\
\hline
\end{tabular}

Table 7: Miller loop cost (see Equation (1)). We assume $a=0$ when $6 \mid k$ and $D=3, b=0$ when $4 \mid k$ and $D=4$, and $a=-3$ otherwise. The second option for $6 \mid k$ is reported by $[5, \S 4]$ to perform slightly better. 


\begin{tabular}{|c|c|c|l|}
\hline$k$ & $\left(p^{k}-1\right) / \Phi_{k}(p)$ & $\mathbf{c}_{\text {FIRSTEXP }}$ & comment \\
\hline 5 & $p-1$ & $(4 \mathbf{m})+\mathbf{i}_{5}+\mathbf{m}_{5}$ & Can omit $\mathbf{f}_{5}=4 \mathbf{m}$ which appears in $\mathbf{i}_{5}$ \\
\hline 6 & $(p+1)\left(p^{3}-1\right)$ & $\begin{array}{c}\mathbf{f}_{6}+\mathbf{m}_{6}+2 \mathbf{s}_{3}+3 \mathbf{m}_{3}+\mathbf{i}_{3} \\
=4 \mathbf{m}+\mathbf{m}_{6}+\mathbf{i}_{6}+\mathbf{m}_{3}\end{array}$ & $\begin{array}{l}\text { Uses } \mathbb{F}_{p^{6}}=\mathbb{F}_{p^{3}}(y)=\mathbb{F}(x)(y) \\
\text { with } y^{2}=x \text { and } x^{3}=\beta\end{array}$ \\
\hline 7 & $p-1$ & $(6 \mathbf{m})+\mathbf{i}_{7}+\mathbf{m}_{7}$ & Can omit $\mathbf{f}_{7}=6 \mathbf{m}$ which appears in $\mathbf{i}_{7}$ \\
\hline 8 & $p^{4}-1$ & $\mathbf{i}_{8}+\mathbf{m}_{8}$ & \\
\hline
\end{tabular}

Table 8: Cost $\mathbf{c}_{\text {FIRSTEXP }}$ of the first part of the final exponentiation

\subsection{Final exponentiation, first and second part}

The final exponentiation to the power $\left(p^{k}-1\right) / r$ is computed in two steps, named first and second part, corresponding to the two factors of the exponent: $\frac{p^{k}-1}{\Phi_{k}(p)} \times \frac{\Phi_{k}(p)}{r}$.

The first part of the exponentiation uses few Frobenius powers and inversions and its cost (Table 8) depends on the value of $\Phi_{k}(p)$. Its computation is very efficient because of Frobenius powers (Remark 3). In particular, for $x \in \mathbb{F}_{p^{8}}, x^{p^{4}}$ is almost free: it is simply the conjugate of $x$ seen in a quadratic extension of $\mathbb{F}_{p^{4}}$.

The second part of the exponentiation is more expensive and is specific to each curve. The key ingredient is the base- $p$ representation of the exponent, since Frobenius powers $p^{i}$ are computed efficiently. Notice that in Algorithm 2, we have $p \equiv(t-1) \equiv\left(t_{0}-1\right) \bmod r$. Let $c$ be such that $p+1-t_{0}=c \cdot r$. The expression $\left(\Phi_{k}(p)-\Phi_{k}\left(t_{0}-1\right)\right) / r$ simplifies, and we obtain a nice generic formula in $p$ and $t_{0}$ for each embedding degree. The actual expression depends on the exponent $i$ in Algorithm 2, as well as on congruence conditions on $T$. We only detail a few examples. Formulas for the other cases can be obtained with the companion software mentioned in $§ 1.1$.

For $k=8$, we choose $D=4$ so that $\sqrt{-D}=2 T^{2}$. When for instance we choose $i=1 \mathrm{in} \mathrm{Algorithm} \mathrm{2,} \mathrm{we} \mathrm{have} t_{0}=T+1 \bmod r$. This leads to the following expression, where $h_{u}$ denotes the integer $\left(h_{t}+1\right) / 2$.

$$
\begin{aligned}
\Phi_{8}(p) / r= & \Phi_{8}\left(t_{0}-1\right) / r+\left(p+t_{0}-1\right)\left(p^{2}+\left(t_{0}-1\right)^{2}\right) c, \\
c= & \left(\left(\left(h_{u}^{2}-h_{u}+h_{y}^{2}+1 / 4\right) T+h_{y}\right) T-h_{y}+1 / 4\right) T \\
& \left.+h_{u}-1\right) T+h_{u}^{2}+h_{y}^{2}
\end{aligned}
$$

where $\Phi_{8}\left(t_{0}-1\right) / r=\Phi_{8}(T) / r=1$ by construction. To raise to the power $\Phi_{8}(p) / r$, we use the fact that $T$ is even to deal with fractional values in the exponent. We obtain the following upper bound on the cost, using $\mathbf{c}_{T}, \mathbf{c}_{u}, \mathbf{c}_{y}$ to denote the cost of raising to the power $T, h_{u}$, and $h_{y}$, respectively:

$$
\mathbf{c}_{\mathrm{SECONDEXP}, k=8}=\left(3 \mathbf{c}_{T}+2 \mathbf{f}_{k}+3 \mathbf{m}_{k}\right)+\left(11 \mathbf{m}_{k}+4 \mathbf{c}_{T}+2 \mathbf{c}_{u}+2 \mathbf{c}_{y}\right) .
$$

For $k=6$, we choose $D=3$ so that $\sqrt{-D}=2 T-1$. We obtain expressions that vary slightly depending on $i$, and on the congruence class of $h_{t} \bmod 2$ and 
$T$ mod 3. It also appears that it is more convenient to compute the cube of the pairing. When for instance we choose $i=1$ in Algorithm 2, and that $T \bmod 3=1$ and $h_{t} \bmod 2=1$, we have the following expression, where $u=\left(h_{t}+1\right) / 2$, $w=h_{y} / 2$, and $T^{\prime}=T-(T \bmod 3)=T-1$ :

$$
\begin{aligned}
3 \Phi_{6}(p) / r= & 3 \Phi_{6}\left(t_{0}-1\right) / r+3\left(p+t_{0}\right) c \\
3 c= & \left(\left(3 u^{2}+9 w^{2}-3 u-3 w+1\right) T^{\prime}+\right. \\
& \left.3 u^{2}+9 w^{2}-6 w\right) T^{\prime}+3 u^{2}+9 w^{2}+3 u-9 w .
\end{aligned}
$$

Raising to the power $3 \Phi_{k}(p) / r$ thus has the following cost (we give an upper bound on all possible congruence conditions). We use $\mathbf{c}_{u}, \mathbf{c}_{w}, \mathbf{c}_{T}$ and $\mathbf{c}_{T^{\prime}}$ to denote the cost of raising to the powers $u, w, T$ and $T^{\prime}=T-(T \bmod 3)$, respectively.

$$
\mathbf{c}_{\mathrm{SECONDEXP}, k=6}=\left(\mathbf{c}_{T}+\mathbf{f}_{k}+2 \mathbf{s}_{k}+4 \mathbf{m}_{k}\right)+\left(12 \mathbf{m}_{k}+2 \mathbf{s}_{k}+2 \mathbf{c}_{u}+2 \mathbf{c}_{w}+2 \mathbf{c}_{T^{\prime}}\right)
$$

For $k=5$ and $k=7$, we use $p=\left(t_{0}-1\right)+c \Phi_{k}(T)$ to reduce $\Phi_{k}(p) / \Phi_{k}(T)$ (rational fraction in the indeterminate $T$ ) to the form

$$
\Phi_{k}(p) / r=\sum_{0 \leq j \leq k-2} p^{j} a_{j}(c, T)
$$

The exact expression of the coefficients $\left(a_{j}\right)_{j}$ depends on $k$ and $i$, and so does the cost of raising to these powers. For example, for $k=5$ and $i=2$, we have

$$
\left(a_{j}\right)_{0 \leq j \leq 3}=\left(-c T^{3}-T+1,-c T^{3}-(c+1) T+1, c T^{2}+c+1, c\right) .
$$

By applying this method, we found that for $k=5$ and $k=7$, raising to the power $\Phi_{k}(p) / r$ costs at most

$$
\mathbf{c}_{\mathrm{SECONDEXP}, k \in\{5,7\}}=2 \mathbf{i}_{k}+(k-2)\left(\mathbf{f}_{k}+\mathbf{c}_{T}+2 \mathbf{m}_{k}\right)+\mathbf{c}_{c}+\mathbf{m}_{k}
$$

where the two inversions can be saved in the favorable case $i=1$, and $\mathbf{c}_{T}$ and $\mathbf{c}_{c}$ are the costs of raising to the powers $T$ and $c$, respectively.

\section{Comparisons with previous curves}

We compare curves generated with Algorithm 2 of embedding degree 5 to 8 with the state of the art: BN and BLS12 curves [3], KSS16 curves [21, §4], and $k=1$ curves [16]. Note that several estimates below differ marginally from [7], which uses a different estimated cost $\mathbf{i}_{12}=\mathbf{i}_{1}+97 \mathbf{m}$, and also reproduce the $7 \mathbf{m}_{2}$ estimate that we mentioned in footnote 4 on page 14 .

BN curve with a 462-bit prime $\boldsymbol{p}$. Barbulescu and Duquesne give in [7] parameters of a BN curve for 128 bits of security. The curve is defined from the parameter $u=2^{114}+2^{101}-2^{14}-1$ and has a prime $p$ of 462 bits (see Table 2). An estimation of the optimal ate pairing on this curve is also given in [7], we 
reproduce the final count. The Miller loop iterates on $T=6 u+2$, of 117 bits and NAF-Hamming-weight 7. Reusing Equation (1) and Tables 6 and 7, and taking into account the correcting terms of the Miller loop for BN curves [52], we get:

$$
\begin{aligned}
\mathbf{c}_{\text {MillerLoop }}= & 116\left(3 \mathbf{m}_{2}+6 \mathbf{s}_{2}+4 \mathbf{m}\right)+115\left(\mathbf{s}_{12}+13 \mathbf{m}_{2}\right) \\
& +6\left(11 \mathbf{m}_{2}+2 \mathbf{s}_{2}+4 \mathbf{m}+13 \mathbf{m}_{2}\right) \\
& +\left(11 \mathbf{m}_{2}+2 \mathbf{s}_{2}+4 \mathbf{m}\right)+4 \mathbf{m}_{2}+4 \mathbf{m}+2\left(13 \mathbf{m}_{2}\right)+4(10 \mathbf{m}) \\
= & 12180 \mathbf{m} .
\end{aligned}
$$

According to [34, Corollary 4.1], raising to the power $u$ costs

$$
\mathbf{c}_{u}=4(114-1) \mathbf{m}_{2}+(6 \cdot 3-3) \mathbf{m}_{2}+3 \mathbf{m}_{12}+3 \cdot 3 \mathbf{s}_{2}+\mathbf{i}_{2}=1585 \mathbf{m}+\mathbf{i} .
$$

The final exponentiation costs

$$
\mathbf{c}_{\text {FinALExp }}=\mathbf{i}_{12}+12 \mathbf{m}_{12}+3 \mathbf{s}_{12}^{\text {cyclo }}+4 \mathbf{f}_{12}+3 \mathbf{c}_{u}=5591 \mathbf{m}+4 \mathbf{i}
$$

where $\mathbf{s}_{12}^{\text {cyclo }}$ is the cost of cyclotomic squarings (see [34]), namely $\mathbf{s}_{12}^{\text {cyclo }}=18 \mathbf{m}$. The optimal ate pairing on the BN-462 curve costs in total $17771 \mathbf{m}+4 \mathbf{i}$.

BLS12 curve with a 461-bit prime $\boldsymbol{p}$. We reproduce the results from [3] adapted to the parameter $u=-2^{77}+2^{50}+2^{33}$ from [7]. We obtain:

$$
\begin{aligned}
\mathbf{c}_{\text {MILLERLOP }}= & 76\left(3 \mathbf{m}_{2}+6 \mathbf{s}_{2}+4 \mathbf{m}\right)+75\left(\mathbf{s}_{12}+13 \mathbf{m}_{2}\right) \\
& +2\left(11 \mathbf{m}_{2}+2 \mathbf{s}_{2}+4 \mathbf{m}+13 \mathbf{m}_{2}\right) \\
= & 7685 \mathbf{m} .
\end{aligned}
$$

As above, we adapt [34, Corollary 4.1]. Raising to the power $u$ costs

$$
\begin{aligned}
\mathbf{c}_{u} & =4(77-1) \mathbf{m}_{2}+(6 \cdot 1-3) \mathbf{m}_{2}+2 \mathbf{m}_{12}+3 \cdot 2 \cdot \mathbf{s}_{2}+\mathbf{i}_{2} \\
& =1045 \mathbf{m}+\mathbf{i} .
\end{aligned}
$$

The final exponentiation costs

$$
\begin{aligned}
\mathbf{c}_{\text {FINALEXP }} & =\mathbf{i}_{12}+12 \mathbf{m}_{12}+2 \mathbf{s}_{12}^{\text {cyclo }}+4 \mathbf{f}_{12}+5 \mathbf{c}_{u} \\
& =6043 \mathbf{m}+6 \mathbf{i} .
\end{aligned}
$$

The optimal ate pairing on the BLS12-461 curve costs in total $13728 \mathbf{m}+6 \mathbf{i}$.

KSS16 curve with a 339-bit prime $\boldsymbol{p}$. We reproduce the results from [21] with the parameter $u=2^{35}-2^{32}-2^{18}+2^{8}+1$ from [7]. We obtain:

$$
\begin{aligned}
\mathbf{c}_{\text {MillerLoop }}= & 34\left(2 \mathbf{m}_{4}+8 \mathbf{s}_{4}+8 \mathbf{m}\right)+33\left(\mathbf{s}_{16}+\left(8 \mathbf{m}_{4}\right)\right)+4\left(9 \mathbf{m}_{4}+5 \mathbf{s}_{4}+8 \mathbf{m}\right) \\
& +3(14 \mathbf{m})+5 \mathbf{m}_{4}+\mathbf{s}_{4}+16 \mathbf{m}+6\left(8 \mathbf{m}_{4}\right) \\
= & 7691 \mathbf{m} .
\end{aligned}
$$


Raising to the power $u$ costs $34 \mathbf{s}_{16}^{\text {cyclo }}+4 \mathbf{m}_{16}=1548 \mathbf{m}$, and the final exponentiation costs:

$$
\begin{aligned}
\mathbf{c}_{\text {FINALEXP }} & =\mathbf{i}_{16}+32 \mathbf{m}_{16}+34 \mathbf{s}_{16}^{\text {cyclo }}+8 \mathbf{f}_{16}+24 \mathbf{m}_{4}+9(1684 \mathbf{m}) \\
& =18210 \mathbf{m}+\mathbf{i} .
\end{aligned}
$$

The optimal ate pairing on the KSS16-339 curve costs in total $25901 \mathbf{m}+\mathbf{i}$.

Curves of embedding degree one. The curves suggested by [16] are resistant to TNFS because the target finite field is $\mathbb{F}_{p}$, with $p$ as large as 3072 bits. The ate pairing is not available on these curves because the trace is $t=2$, so the Tate pairing must be used. Its cost is given in [16]: for a 256-bit $r$, the Miller loop costs $4626 \mathbf{m}+\mathbf{i}$ and the final exponentiation costs $4100 \mathbf{m}$. The total cost is finally $8726 \mathrm{~m}+\mathbf{i}$.

\subsection{Our new STNFS-resistant curves at the 128-bit security level}

We generate four curves of embedding degree 5, 6, 7 and 8 with Algorithm 2 combined with the CM method and estimate the cost of the new optimal ate pairing on these curves. Code to reproduce this search can be found in the repository mentioned in $\S 1.1$.

Twist-secure and subgroup-secure parameters. We checked our curves for twistand subgroup-security (see [10]). For each curve, we checked the size of the cofactors of the curve and its quadratic twist on $\mathbb{G}_{1}$ and $\mathbb{G}_{2}$. A curve $E$ is $\eta$ subgroup-secure over $\mathbb{F}_{q}$ if all the factors of $E\left(\mathbb{F}_{q}\right)$ are at least as large as $r$, except those of size $\eta$. A curve is twist-subgroup secure if its quadratic twist is subgroup-secure. This makes five criteria: subgroup- and twist-subgroup- security for both $\mathbb{G}_{1}$ and $\mathbb{G}_{2}$, as well as subgroup security for $\mathbb{G}_{T}$ (with respect to $\Phi_{k}(p)$ ). We selected four curves that are subgroup and twist-subgroup secure for $\mathbb{G}_{1}$ with $\eta=13$. Except in the $k=7$ case, the curve containing the subgroup $\mathbb{G}_{2}$ is also subgroup-secure. For embedding degree 5 and 6 , this curve is also twist-subgroupsecure. We did not investigate the $\mathbb{G}_{T}$ subgroup-security: together with the Cocks-Pinch conditions, it would require finding parameters such that $\Phi_{k}(p) / r$ is prime or almost prime. With the sizes provided in the following paragraph, $1088 \leq \log _{2}\left(\Phi_{k}(p) / r\right) \leq 2816$ so it is difficult to factor this thousand-bit integer entirely and it will very unlikely be prime.

Parameter choices. We explain our choices of parameter sizes in Algorithm 2:

Size of the prime $p$ We target a size for the finite field $\mathbb{F}_{p^{k}}$ that determines the size of $p$. These values can be read in Table 5 .

Hamming weight (or 2-NAF weight) of $T$ We restrict to low weight $T$ in order to get an efficient Miller loop. We choose $\mathrm{HW}_{2-\mathrm{NAF}}(T)=4$ for the $k=5$ curve, or $\mathrm{HW}_{2-\mathrm{NAF}}(T)=5$ for others. 
Discriminant For efficiency, we target curves with as many automorphisms as possible. For $k=6$ (resp. 8), we set $D=3$ (resp. 4) so that a sextic (resp. quartic) twist is available. For $k=5$, we chose arbitrarily $D \approx 10^{10}$, which is well within the feasible range for the CM method. For $k=7$, the size of $p$ (512 bits) restricts us to small discriminants, since we must have $4 p=t^{2}+D y^{2}$ with $\log _{2}(t), \log _{2}(y) \approx \log _{2}(r)=256$.

Hamming weight (or 2-NAF weight) of $h_{t}$ and $h_{y}$ As explained in $\S 5.2$, for $k=6$ and $k=8$ we restrict to low weight cofactors $h_{t}$ and $h_{y}$ so as to accelerate the exponentiation to the power $c$ in the second part of the final exponentiation (see Equations (2) and (3)).

Allowing a cofactor of 13 bits, we obtain twist- and subgroup-secure elliptic curves of embedding degree five to eight. We denote by $E^{t}$ the quadratic twist of $E$ and $\tilde{E}$ the degree $d$ twist of $E$ such that $E\left(\mathbb{F}_{p^{k}}\right)[r] \simeq \tilde{E}\left(\mathbb{F}_{p^{k / d}}\right)[r]$. Points on $E$ can be represented with the Edwards model if we restrict to curves with 4 -torsion points. For the remainder of this section, the notation $p_{N}$ denotes an arbitrary prime of $N$ bits.

Curve of embedding degree 5 The curve $E: y^{2}=x^{3}-3 x+b_{5}$ defined over $\mathbb{F}_{p}$ with

$$
\begin{aligned}
b_{5}= & 0 x 3 d d 2 d 2 b 0 b 2 e 68770 b f 01 b 41946 a b 867390 c f 9 e c c 4 a 858004 f c 769 c \\
& 278 f 079574677 c 7 d b 3 e 7201 c 938 b 099 f 85 e b 6 e 85 f 200 b 95 a 80 b 24 f d b \\
& d f 584098 d 690 c 6 b 91 b 21 d 00 f 52 c c 79473 a 11123 b 08 a b 2 a 616 b 4 a 4 f b f \\
p= & 0 x 40000138 c d 26 a b 94 b 86 e 1 b 2 f 7482785 f a 18 f 877591 d 2 a 4476 b 4760 \\
& 217 f 860 b f e 8674 e 2 a 4610 d 669328 b d a 13044 c 030 e 8 c c 836 a 5 b 363 f 2 d \\
& 4 c 8 a b c a b 71 b 12091356 b b 4695 c 5626 b c 319 d 38 b f 65768 c 5695 f 9 a d 97
\end{aligned}
$$

satisfies

$$
\begin{aligned}
& \# E\left(\mathbb{F}_{p}\right)=2^{2} \cdot p_{405} \cdot r \quad \# \tilde{E}\left(\mathbb{F}_{p^{5}}\right)=p_{2393} \cdot\left(2^{2} \cdot p_{405} \cdot r\right) \cdot r \\
& \# E^{t}\left(\mathbb{F}_{p}\right)=2^{2} \cdot p_{661} \quad \# E^{t}\left(\mathbb{F}_{p^{5}}\right)=p_{2649} \cdot\left(2^{2} \cdot p_{661}\right) \\
& r=0 \times 9610000000015700 \mathrm{ab} 80000126012600 \mathrm{c} 4007000 \mathrm{a} 800 \mathrm{e} 000 \mathrm{f} 000200040008001
\end{aligned}
$$

The additional parameters to obtain the curve from Algorithm 2 are :

$$
T=2^{64}-2^{61}+2^{15}, D=10^{10}+147, i=1, h_{t}=3, h_{y}=0 \mathrm{x} 11 \mathrm{e} 36418 \mathrm{c} 7 \mathrm{c} 8 \mathrm{~b} 454
$$

and $\mathbb{F}_{p^{5}}$ can be defined as $\mathbb{F}_{p}[x] /\left(x^{5}-5\right)$.

Curve of embedding degree 6 The curve $E: y^{2}=x^{3}-1$ defined over $\mathbb{F}_{p}$ with

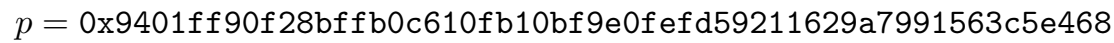

$$
\begin{aligned}
& \text { d43ec9cfe1549fd59c20ab5b9a7cda7f27a0067b8303eeb4b31555cf } 4 \\
& \text { f24050ed155555cd7fa7a5f8aaaaaaad47ede1a6aaaaaaab69e6dcb }
\end{aligned}
$$


satisfies

$$
\begin{array}{cl}
\# E\left(\mathbb{F}_{p}\right)=2^{2} \cdot p_{414} \cdot r & \# \tilde{E}\left(\mathbb{F}_{p}\right)=3 \cdot p_{414} \cdot r \\
\# E^{t}\left(\mathbb{F}_{p}\right)=2^{2} \cdot 3 \cdot 7 \cdot p_{665} & \# E^{t}\left(\mathbb{F}_{p}\right)=13 \cdot 19 \cdot p_{664}
\end{array}
$$

$r=0$ xe0ffffffffffffc400000000000003ff 10000000000000200000000000000001

The additional parameters to obtain the curve from Algorithm 2 are :

$$
T=2^{128}-2^{124}-2^{69}, D=3, i=1, h_{t}=-1, h_{y}=2^{80}-2^{70}-2^{66}-0 \times 3 \mathrm{fe} 0
$$

and $\mathbb{F}_{p^{6}}$ can be defined as $\mathbb{F}_{p}[x] /\left(x^{6}-2\right)$.

Curve of embedding degree 7 The curve $E: y^{2}=x^{3}-3 u^{2} x+b_{7} u^{3}$ defined over $\mathbb{F}_{p}$ with

$$
\begin{aligned}
b_{7}= & 0 x 15 \mathrm{~d} 384 \mathrm{c} 76889 \mathrm{~d} 377 \mathrm{dd} 63600 \mathrm{fbe} 42628 \mathrm{e} 0 \mathrm{c} 386 \mathrm{a} 3 \mathrm{e} 87 \\
& 915790188 \mathrm{~d} 944845 \mathrm{aab} 2 \mathrm{~b} 649964 \mathrm{f} 386 \mathrm{dc} 90 \mathrm{~b} 3 \mathrm{a} 9 \mathrm{~b} 612 \\
& \text { 0af5da9a2aaead5e415dd958c5cfa80ea61aac268b0 } \\
p= & 0 x 8 f 591 \mathrm{a} 9876 \mathrm{a} 6 \mathrm{~d} 2344 \mathrm{ae} 66 \mathrm{dd} 7540 \mathrm{ea} 2 \mathrm{fd} 28174755 \mathrm{~d} 1 \\
& 6 \mathrm{c} 4 \mathrm{ae} 5 \mathrm{c} 5 \mathrm{~cd} 5 \mathrm{c} 1 \mathrm{~d} 208 \mathrm{e} 639271 \mathrm{~b} 48 \mathrm{c} 8 \mathrm{ba} 7453 \mathrm{c} 95 \mathrm{a} 2 \mathrm{a} 9 \mathrm{~b} \\
& \text { e6434f } 2455504 \mathrm{~d} 419 \mathrm{f} 13 \mathrm{e} 35062 \mathrm{aa} 5 \mathrm{ebbc49ecfd} 30 \mathrm{f} 9 \\
u= & 11
\end{aligned}
$$

satisfies

$$
\# E\left(\mathbb{F}_{p}\right)=2^{2} \cdot 3^{2} \cdot p_{251} \cdot r \quad \# E^{t}\left(\mathbb{F}_{p^{7}}\right)=2^{5} \cdot 5 \cdot p_{504}
$$

$r=0 x b 63 c c d 541 c 3 a a 13 c 7 b 7098 f$ eb312eecf5648fd215c0d2916714b429d14e8f 889

The additional parameters to obtain the curve from Algorithm 2 are :

$$
T=2^{43}-2^{41}-0 \mathrm{x} 47 \mathrm{dfdb} 8, D=20, i=6, h_{t}=-2, h_{y}=0
$$

and $\mathbb{F}_{p^{7}}$ can be defined as $\mathbb{F}_{p}[x] /\left(x^{7}-2\right)$.

Curve of embedding degree 8 The curve $E: y^{2}=x^{3}+2 x$ defined over $\mathbb{F}_{p}$ with

$$
\begin{aligned}
p= & \text { 0xbb9dfd549299f } 1 \mathrm{c} 803 \mathrm{ddd} 5 \mathrm{~d} 7 \mathrm{c05e} \text { cc0373d9b1ac15b } \\
& 47 \mathrm{aa} 5 \mathrm{aa} 84626 \mathrm{f} 33 \mathrm{e} 58 \mathrm{fe} 66943943049031 \mathrm{ae} 4 \mathrm{ca} 1 \mathrm{~d} 2719 \mathrm{~b} \\
& 3 \mathrm{a} 84 \mathrm{fa} 363 \mathrm{bcd} 2539 \mathrm{a} 5 \mathrm{~cd} 02 \mathrm{c} 6 \mathrm{f} 4 \mathrm{~b} 6 \mathrm{~b} 645 \mathrm{a} 58 \mathrm{c} 1085 \mathrm{e} 14411
\end{aligned}
$$

satisfies

$$
\begin{gathered}
\# E\left(\mathbb{F}_{p}\right)=2^{2} \cdot 3^{2} \cdot 5 \cdot 41 \cdot p_{275} \cdot r \quad \# E^{t}\left(\mathbb{F}_{p}\right)=2^{4} \cdot p_{540} \quad \# \tilde{E}\left(\mathbb{F}_{p^{2}}\right)=2 \cdot 89 \cdot p_{824} \cdot r \\
r=\text { 0xff0060739e18d7594a978b0ab6ae4ce3dbfd52a9d00197603fffdf0000000101 }
\end{gathered}
$$

The additional parameters to obtain the curve from Algorithm 2 are :

$$
T=2^{64}-2^{54}+2^{37}+2^{32}-4, D=4, i=1, h_{t}=1, h_{y}=0 x \operatorname{xdc} 04
$$

and $\mathbb{F}_{p^{8}}$ can be defined as $\mathbb{F}_{p}[x] /\left(x^{8}-5\right)$. 


\subsection{Comparison with the state of the art}

In order to compare the pairing on our curves with the computation on $\mathrm{BN}$, BLS12, KSS16, and $k=1$ curves, we need to determine the cost of a multiplication m for different sizes of $p$. Indeed, a multiplication in the 3072-bit field of $k=1$ curves is much more expensive than in a 512-bit prime field. Table 9 shows the benchmarks with RELIC [4] for base field arithmetic with the different primes involved in our pairings.

Pairing computation. The costs of the Miller loop and the first part of the final exponentiation are given by Equation (1), Table 7, and Table 8. The second part of the final exponentiation is covered by $\S 5.2$. This part is specific to each set of curve parameters, in particular $\mathrm{HW}_{2-\mathrm{NAF}}\left(h_{t}\right)$ and $\mathrm{HW}_{2-\mathrm{NAF}}\left(h_{y}\right)$. Appendix $\mathrm{A}$ goes into more detail for our curve with $k=8$. Further detail for the second part of the final exponentiation for all exponents, covering the various cases, can be found in the code repository (see $§ 1.1$ ).

Table 10 summarises our comparison results for pairing computations. We warn the reader that timings of Table 10 are not real pairing computations, as we simply used as a base the arithmetic operations of RELIC [4], and the multiplication costs that we detailed in the paragraphs above. This being said, for the curves where an actual implementation of the optimal ate pairing is available with RELIC (BN and BLS12 curves), the estimation that we obtain is within $10 \%$ of the actual computation time. This gives reasonable confidence for the validity of the other projected timings.

Miller loop. We obtain a faster Miller loop for $k=6$ and $k=8$ curves compared to BN and BLS12 curves. The $k=8$ curve has a shorter Miller loop (64-bit) compared to the BN and BLS12 ones (117-bit). The $k=6$ curve has a sextic twist that allows to compute $f_{T, Q}(P)$ on $\mathbb{F}_{p}$ of 672 bits, compared to a quadratic field of 922 bits for BN and BLS12 curves. As for the cases $k=5$ and $k=7$, the Miller loop is not as efficient because no twist is available, and the computation is done over $\mathbb{F}_{p^{k}}$. Comparisons between $k=6$ and $k=7$ curves show that using a curve with twists is a better option than having a short Miller loop. The best option is obviously to have a short Miller loop and a curve with twists, as for $k=8$ curves.

Final exponentiation. The rewriting tricks used in $\S 5.2$ for the final exponentiation apply for any curve obtained with Algorithm 2 with the optimisation $r=\Phi_{k}(T)$. For $k=6$ and $k=8$ the cofactor is smaller, and the discriminant $D=3$, resp. $D=4$, gives formulas that are as good as for BN and BLS12 curves. For $k=5$ and $k=7$ curves, the exponentiation is less efficient because fast cyclotomic squaring formulas are not available.

Total cost. Table 10 shows that our new pairing is almost as efficient as the optimal ate pairing on the BLS12 and KSS16 curves. Given the nature of Table 10 which gives estimated timings, it is however more appropriate to say that the 
performance difference is within the error margin. Additionally, we estimate that the optimal ate pairing on our $k=8$ curve is up to 22 times more efficient than the Tate pairing on $k=1$ curves [16].

Remark 4. We also estimated the cost of a Tate pairing on Cocks-Pinch curves without twists for $k=5,7$ where the first input point $P$ has coordinates in $\mathbb{F}_{p}$ and the second input point $Q$ has coordinates in $\mathbb{F}_{p^{k}}$. The doublings and additions of points now take place in $\mathbb{F}_{p}$, but the accumulations still require $\mathbb{F}_{p^{k}}$ arithmetic. The costs are $\mathbf{c}_{\text {AddLine }}=8 \mathbf{m}+3 \mathbf{s}+2 k \mathbf{m}, \mathbf{c}_{\text {DoubleLine }}=7 \mathbf{m}+4 \mathbf{s}+2 k \mathbf{m}$, $\mathbf{c}_{\text {Verticalline }}=k \mathbf{m}, \mathbf{c}_{\text {Update } 1}=2 \mathbf{m}_{k}+\mathbf{s}_{k}$, and $\mathbf{c}_{\text {Update } 2}=2 \mathbf{m}_{k}\left(m_{d} \in \mathbb{F}_{p}\right.$ is not computed since it would be 1 after the final exponentiation). The Miller length is $(k-1)$ times longer, of length $\log _{2} r$ instead of $\log _{2} T$. The accumulation steps $\mathbf{c}_{\text {UPdate } 1}$ cost $(k-1)$ times more. The Miller loop of a Tate pairing would cost roughly $\left(\log _{2}(r)-1\right)\left(\mathbf{c}_{\text {DoubleLine }}+\mathbf{c}_{\text {Verticalline }}\right)+\left(\log _{2}(r)-2\right) \mathbf{c}_{\text {Update } 1}+$ $\left(\mathrm{HW}_{2-\mathrm{NAF}}(r)-1\right)\left(\mathbf{c}_{\mathrm{AdDL}} \mathrm{ine}+\mathbf{c}_{\text {VerticalLine }}+\mathbf{c}_{\mathrm{Update} 2}\right)+\mathbf{i}_{k}$. For $k=5$ we have $\log _{2}(r)=256$ and $\mathrm{HW}_{2-\mathrm{NAF}}(r)=39$, which gives $18585 \mathrm{~m}$. For $k=7$ we have $\log _{2}(r)=256$ and $\mathrm{HW}_{2-\mathrm{NAF}}(r)=90$, which gives $31817 \mathbf{m}$. The gain of swapping $P$ and $Q$ in the Tate pairing is counterbalanced by the much longer Miller loop and finally, the Miller loop of a Tate pairing would require more multiplications in $\mathbb{F}_{p}$ compared to an optimal ate Miller loop costing $14496 \mathbf{m}$ for $k=5$ and $18830 \mathrm{~m}$ for $k=7$ (see Table 10).

\begin{tabular}{|c|c|c|}
\hline Prime size & Building block for & $\mathbb{F}_{p}$ multiplication \\
\hline $192<\log _{2}(p) \leq 256$ & & $32 \mathrm{~ns}$ \\
\hline $320<\log _{2}(p) \leq 384$ & KSS16 & $65 \mathrm{~ns}$ \\
\hline $384<\log _{2}(p) \leq 448$ & BN, BLS12 & $85 \mathrm{~ns}$ \\
\hline $448<\log _{2}(p) \leq 512$ & BN, BLS12, $k=7$ & $106 \mathrm{~ns}$ \\
\hline $512<\log _{2}(p) \leq 576$ & $k=8$ & $129 \mathrm{~ns}$ \\
\hline $576<\log _{2}(p) \leq 640$ & & $154 \mathrm{~ns}$ \\
\hline $640<\log _{2}(p) \leq 704$ & $k=5, k=6$ & $181 \mathrm{~ns}^{*}$ \\
\hline $3008<\log _{2}(p) \leq 3072$ & {$[16]$} & $3800 \mathrm{~ns}^{* *}$ \\
\hline
\end{tabular}

Table 9: $\mathbb{F}_{p}$ multiplication timing for RELIC on a Intel Core i7-8700 CPU, $3.20 \mathrm{GHz}$ with TurboBoost disabled

*Estimation because no bench is available for 11 machine words primes.

**Benched with GNU MP

Elliptic curve scalar multiplication in $\mathbb{G}_{1}$ and $\mathbb{G}_{2}$. Our generation of curve leads to large prime value (up to eleven 64-bit words instead of eight for BN and BLS12 curves). The scalar multiplication cost on $\mathbb{G}_{1}$ is not affected by slow finite field multiplications because our curves benefit of other improvements: BN (resp. BLS) curves parameters for 128 bits of security lead to scalar multiplications $[k] P$ on $\mathbb{G}_{1}$ and $\mathbb{G}_{2}$ with $\log _{2}(k) \approx 448$ (resp. 300). For our curves of embedding 


\begin{tabular}{|c|c|c|c|c|c|}
\hline Curve & Prime & $\begin{array}{l}\text { Miller loop } \\
\text { time estimation }\end{array}$ & $\begin{array}{c}\text { Exponentiation } \\
\text { time estimation }\end{array}$ & Total & $\begin{array}{c}\text { time } \\
\text { estimation }\end{array}$ \\
\hline$k=5$ & 663-bit & $\begin{array}{c}14496 \mathrm{~m} \\
2.6 \mathrm{~ms}\end{array}$ & $\begin{array}{c}9809 \mathrm{~m} \\
1.8 \mathrm{~ms}\end{array}$ & $24305 \mathbf{m}$ & $4.4 \mathrm{~ms}$ \\
\hline$k=6$ & 672-bit & $\begin{array}{c}4601 \mathbf{m} \\
0.8 \mathrm{~ms}\end{array}$ & $\begin{array}{c}3871 \mathrm{~m} \\
0.7 \mathrm{~ms}\end{array}$ & $8472 \mathrm{~m}$ & $1.5 \mathrm{~ms}$ \\
\hline$k=7$ & 512-bit & $\begin{array}{c}18330 \mathrm{~m} \\
1.9 \mathrm{~ms}\end{array}$ & $\begin{array}{c}13439 \mathrm{~m} \\
1.4 \mathrm{~ms}\end{array}$ & $31769 \mathbf{m}$ & $3.4 \mathrm{~ms}$ \\
\hline$k=8$ & 544-bit & $\begin{array}{l}4502 \mathrm{~m} \\
0.6 \mathrm{~ms}\end{array}$ & $\begin{array}{c}7056 \mathrm{~m} \\
0.9 \mathrm{~ms}\end{array}$ & $11558 \mathbf{m}$ & $1.5 \mathrm{~ms}$ \\
\hline $\mathrm{BN}$ & 446-bit & $\begin{array}{c}11620 \mathrm{~m} \\
1.0 \mathrm{~ms}\end{array}$ & $\begin{array}{c}5349 \mathrm{~m} \\
0.5 \mathrm{~ms}\end{array}$ & $16969 \mathbf{m}$ & $1.4 \mathrm{~ms}$ \\
\hline BLS12 & 446-bit & $\begin{array}{c}7805 \mathrm{~m} \\
0.7 \mathrm{~ms}\end{array}$ & $\begin{array}{c}7723 \mathrm{~m} \\
0.7 \mathrm{~ms}\end{array}$ & $15528 \mathbf{m}$ & $1.3 \mathrm{~ms}$ \\
\hline $\mathrm{BN}$ & 462-bit & $\begin{array}{c}12180 \mathrm{~m} \\
1.3 \mathrm{~ms}\end{array}$ & $\begin{array}{c}5727 \mathbf{m} \\
0.6 \mathrm{~ms} \\
\end{array}$ & $17907 \mathbf{m}$ & $1.9 \mathrm{~ms}$ \\
\hline BLS12 & 461-bit & $\begin{array}{c}7685 \mathrm{~m} \\
0.8 \mathrm{~ms}\end{array}$ & $\begin{array}{c}6283 \mathrm{~m} \\
0.7 \mathrm{~ms}\end{array}$ & $13968 \mathbf{m}$ & $1.5 \mathrm{~ms}$ \\
\hline KSS16 & 339-bit & $\begin{array}{c}7691 \mathrm{~m} \\
0.5 \mathrm{~ms}\end{array}$ & $\begin{array}{c}18235 \mathrm{~m} \\
1.2 \mathrm{~ms}\end{array}$ & $25926 \mathbf{m}$ & $1.7 \mathrm{~ms}$ \\
\hline$k=1$ & 3072-bit & $\begin{array}{l}4651 \mathrm{~m} \\
17.7 \mathrm{~ms}\end{array}$ & $\begin{array}{l}4100 \mathrm{~m} \\
15.6 \mathrm{~ms}\end{array}$ & $8751 \mathrm{~m}$ & $33.3 \mathrm{~ms}$ \\
\hline
\end{tabular}

Table 10: Pairing cost and timing extrapolation from Table 9

degree five to eight, we choose $r$ of minimal size (256 bits to withstand the Pollard rho attack). Some curves get benefits of efficient group law arithmetic: curves of embedding degree 5, 7, and 8 use the Edwards model. The $k=6$ curve use the efficient formulas available for $a=0$ curves, widely used in practice. The Gallant-Lambert-Vanstone (GLV) method can be performed on $k=6$ and $k=8$ curves in order to reduce the number of doubling and addition steps. Over $\mathbb{G}_{2}$, the scalar multiplication is often accelerated by using a twist of the curve. The trick is available for curves of degree 6 and 8 , but not for $k=5$ and 7 . Even if the main topic of this paper is about pairing computations, various protocols also compute scalar multiplications. Curves of embedding degree 5 and 7 do not benefit of twists and GLV optimisation, so the cost over $\mathbb{G}_{2}$ is too expensive for practical applications.

\section{Conclusion}

We modified the Cocks-Pinch method to generate pairing-friendly elliptic curves with an optimal Miller loop length $\log r / \varphi(k)$ to compute efficiently an optimal ate pairing. Moreover the parameters are carefully chosen so that the curves withstand the recent STNFS attacks on discrete logarithms. Our projected timings for optimal ate pairing computation on our $k=6$ and $k=8$ curve seems to be close to the BN or BLS12 curves, and the difference is very probably within 
the error margin between this projected analysis and an actual impementation. Compared to $k=1$ curves presented in [16], pairing computations on the curves suggested here are expected to be $7(k=5)$ to $22(k=8)$ times faster.

One lesson of our work is that the Miller loop length is very important for an efficient pairing, even in the Cocks-Pinch case. It matters much more than the $\rho$ value.

With respect to the threats on pairing summarised in Table 1, users fearing the progress of NFS variants should prefer the more conservative choice of our modified Cocks-Pinch curves: unlike BN, BLS12, and KSS16 curves, we do not have to use much larger parameters to be STNFS-resistant.

We finally note that the short Miller loop on our $k=6$ and $k=8$ curves is well-suited to protocols where the product of several pairings is computed, the final exponentiation being computed only once, after the Miller loops. This is the case for the translation in the prime-order setting of the Boneh-Boyen IBE scheme: the product of six pairings is computed in the decryption step, and for the hierarchical identity-based encryption based on Lewko-Waters scheme: the product of ten pairings is computed in the decryption step [40].

Acknowledgements. The second author thanks P. Zimmermann for his help with Pari/Gp, and P. Gaudry and T. Kleinjung for their contribution to Table 12.

\section{References}

1. Aoki, K., Franke, J., Kleinjung, T., Lenstra, A.K., Osvik, D.A.: A kilobit special number field sieve factorization. In: Kurosawa, K. (ed.) ASIACRYPT 2007. LNCS, vol. 4833, pp. 1-12. Springer, Heidelberg (Dec 2007). https://doi.org/10.1007/ 978-3-540-76900-2_1

2. Aranha, D.F.: Pairings are not dead, just resting (nov 2017), slides at ECC 2017 workshop. https://ecc2017.cs.ru.nl/

3. Aranha, D.F., Fuentes-Castañeda, L., Knapp, E., Menezes, A., Rodríguez-Henríquez, F.: Implementing pairings at the 192-bit security level. In: Abdalla, M., Lange, T. (eds.) PAIRING 2012. LNCS, vol. 7708, pp. 177-195. Springer, Heidelberg (May 2013). https://doi.org/10.1007/978-3-642-36334-4_11

4. Aranha, D.F., Gouvêa, C.P.L.: RELIC is an Efficient LIbrary for Cryptography. https://github.com/relic-toolkit/relic

5. Aranha, D.F., Karabina, K., Longa, P., Gebotys, C.H., López-Hernández, J.C.: Faster explicit formulas for computing pairings over ordinary curves. In: Paterson, K.G. (ed.) EUROCRYPT 2011. LNCS, vol. 6632, pp. 48-68. Springer, Heidelberg (May 2011). https://doi.org/10.1007/978-3-642-20465-4_5

6. Bai, S., Gaudry, P., Kruppa, A., Thomé, E., Zimmermann, P.: Factorization of RSA220. Number Theory list (May 12 2016), https://listserv.nodak.edu/cgi-bin/ wa. exe?A2=NMBRTHRY; d17fe291.1605, http://www.loria.fr/ zimmerma/papers/ rsa220.pdf

7. Barbulescu, R., Duquesne, S.: Updating key size estimations for pairings. Journal of Cryptology 32(4), 1298-1336 (Oct 2019). https://doi.org/10.1007/ s00145-018-9280-5 
8. Barbulescu, R., Gaudry, P., Guillevic, A., Morain, F.: Improving NFS for the discrete logarithm problem in non-prime finite fields. In: Oswald, E., Fischlin, M. (eds.) EUROCRYPT 2015, Part I. LNCS, vol. 9056, pp. 129-155. Springer, Heidelberg (Apr 2015). https://doi.org/10.1007/978-3-662-46800-5_6

9. Barbulescu, R., Gaudry, P., Kleinjung, T.: The tower number field sieve. In: Iwata, T., Cheon, J.H. (eds.) ASIACRYPT 2015, Part II. LNCS, vol. 9453, pp. 31-55. Springer, Heidelberg (Nov/ Dec 2015). https://doi.org/10.1007/ 978-3-662-48800-3_2

10. Barreto, P.S.L.M., Costello, C., Misoczki, R., Naehrig, M., Pereira, G.C.C.F., Zanon, G.: Subgroup security in pairing-based cryptography. In: Lauter, K.E., RodríguezHenríquez, F. (eds.) LATINCRYPT 2015. LNCS, vol. 9230, pp. 245-265. Springer, Heidelberg (Aug 2015). https://doi .org/10.1007/978-3-319-22174-8_14

11. Bitansky, N., Canetti, R., Chiesa, A., Tromer, E.: From extractable collision resistance to succinct non-interactive arguments of knowledge, and back again. In: Goldwasser, S. (ed.) ITCS 2012. pp. 326-349. ACM (Jan 2012). https: //doi.org/10.1145/2090236.2090263

12. Boneh, D., Franklin, M.K.: Identity-based encryption from the Weil pairing. In: Kilian, J. (ed.) CRYPTO 2001. LNCS, vol. 2139, pp. 213-229. Springer, Heidelberg (Aug 2001). https://doi.org/10.1007/3-540-44647-8_13

13. Boneh, D., Lynn, B., Shacham, H.: Short signatures from the Weil pairing. In: Boyd, C. (ed.) ASIACRYPT 2001. LNCS, vol. 2248, pp. 514-532. Springer, Heidelberg (Dec 2001). https://doi .org/10.1007/3-540-45682-1_30

14. Bouvier, C., Gaudry, P., Imbert, L., Jeljeli, H., Thomé, E.: Discrete logarithms in $\operatorname{GF}(p)-180$ digits. Number Theory list, item 004703 (June 11 2014), https: //listserv . nodak. edu/cgi-bin/wa. exe?A2=NMBRTHRY;615d922a .1406

15. Bowe, S.: BLS12-381: New zk-SNARK elliptic curve construction. Zcash blog (March 11 2017), https://blog.z.cash/new-snark-curve/

16. Chatterjee, S., Menezes, A., Rodríguez-Henríquez, F.: On instantiating pairing-based protocols with elliptic curves of embedding degree one. IEEE Trans. Computers 66(6), 1061-1070 (2017). https://doi.org/10.1109/TC. 2016.2633340

17. Childers, G.: Factorization of a 1061-bit number by the special number field sieve. Cryptology ePrint Archive, Report 2012/444 (2012), http://eprint.iacr.org/ 2012/444

18. Chuengsatiansup, C., Martindale, C.: Pairing-friendly twisted hessian curves. In: Chakraborty, D., Iwata, T. (eds.) INDOCRYPT 2018. LNCS, vol. 11356, pp. 228-247. Springer, Heidelberg (Dec 2018). https ://doi .org/10.1007/978-3-030-05378-9_ 13

19. Chung, J., Hasan, M.A.: Asymmetric squaring formulae. In: 18th IEEE Symposium on Computer Arithmetic (ARITH '07). pp. 113-122 (June 2007). https://doi. org/10.1109/ARITH. 2007.11

20. Cohen, H., Miyaji, A., Ono, T.: Efficient elliptic curve exponentiation using mixed coordinates. In: Ohta, K., Pei, D. (eds.) ASIACRYPT'98. LNCS, vol. 1514, pp. 51-65. Springer, Heidelberg (Oct 1998). https : //doi .org/10.1007/3-540-49649-1_6

21. Costello, C., Lange, T., Naehrig, M.: Faster pairing computations on curves with high-degree twists. In: Nguyen, P.Q., Pointcheval, D. (eds.) PKC 2010. LNCS, vol. 6056, pp. 224-242. Springer, Heidelberg (May 2010). https://doi.org/10. 1007/978-3-642-13013-7_14

22. Devegili, A.J., Ó hÉigeartaigh, C., Scott, M., Dahab, R.: Multiplication and squaring on pairing-friendly fields. Cryptology ePrint Archive, Report 2006/471 (2006), http://eprint.iacr.org/2006/471 
23. Duquesne, S., El Mrabet, N., Fouotsa, E.: Efficient computation of pairings on jacobi quartic elliptic curves. J. Mathematical Cryptology 8(4), 331-362 (2014), https: //doi.org/10.1515/jmc-2013-0033, https://eprint.iacr.org/2013/597

24. Fotiadis, G., Konstantinou, E.: TNFS resistant families of pairing-friendly elliptic curves. Theoretical Computer Science 800, 73-89 (31 December 2019). https: //doi.org/10.1016/j.tcs.2019.10.017, https://eprint.iacr.org/2018/1017

25. Fotiadis, G., Martindale, C.: Optimal TNFS-secure pairings on elliptic curves with even embedding degree. Cryptology ePrint Archive, Report 2018/969 (2018), https://eprint.iacr.org/2018/969

26. Freeman, D., Scott, M., Teske, E.: A taxonomy of pairing-friendly elliptic curves. Journal of Cryptology 23(2), 224-280 (Apr 2010). https://doi.org/10.1007/ s00145-009-9048-z

27. Fried, J., Gaudry, P., Heninger, N., Thomé, E.: A kilobit hidden SNFS discrete logarithm computation. In: Coron, J., Nielsen, J.B. (eds.) EUROCRYPT 2017, Part I. LNCS, vol. 10210, pp. 202-231. Springer, Heidelberg (Apr / May 2017). https://doi.org/10.1007/978-3-319-56620-7_8

28. Gordon, D.M.: Designing and detecting trapdoors for discrete log cryptosystems. In: Brickell, E.F. (ed.) CRYPTO'92. LNCS, vol. 740, pp. 66-75. Springer, Heidelberg (Aug 1993). https://doi.org/10.1007/3-540-48071-4_5

29. Granger, R., Scott, M.: Faster squaring in the cyclotomic subgroup of sixth degree extensions. In: Nguyen, P.Q., Pointcheval, D. (eds.) PKC 2010. LNCS, vol. 6056, pp. 209-223. Springer, Heidelberg (May 2010). https://doi.org/10. 1007/978-3-642-13013-7_13

30. Guillevic, A.: Simulating DL computation in $\operatorname{GF}\left(p^{n}\right)$ with the new variants of the Tower-NFS algorithm to deduce security level estimates (Nov 2017), slides at ECC 2017 workshop. https://ecc2017.cs.ru.nl/

31. Joux, A.: A one round protocol for tripartite Diffie-Hellman. Journal of Cryptology 17(4), 263-276 (Sep 2004). https://doi.org/10.1007/s00145-004-0312-y

32. Joux, A., Lercier, R.: Improvements to the general number field sieve for discrete logarithms in prime fields. A comparison with the Gaussian integer method. Math. Comp. 72(242), 953-967 (2003). https://doi.org/10.1090/S0025-5718-02-01482-5

33. Joux, A., Pierrot, C.: The special number field sieve in $\mathbb{F}_{p^{n}}$ - application to pairing-friendly constructions. In: Cao, Z., Zhang, F. (eds.) PAIRING 2013. LNCS, vol. 8365, pp. 45-61. Springer, Heidelberg (Nov 2014). https://doi.org/10.1007/ 978-3-319-04873-4_3

34. Karabina, K.: Squaring in cyclotomic subgroups. Math. Comput. 82(281), 555-579 (2013). https://doi.org/10.1090/S0025-5718-2012-02625-1

35. Khandaker, M.A.A., Nanjo, Y., Ghammam, L., Duquesne, S., Nogami, Y., Kodera, Y.: Efficient optimal ate pairing at 128-bit security level. In: Patra, A., Smart, N.P. (eds.) INDOCRYPT 2017. LNCS, vol. 10698, pp. 186-205. Springer, Heidelberg (Dec 2017). https://doi.org/10.1007/978-3-319-71667-1_10

36. Kim, T., Barbulescu, R.: Extended tower number field sieve: A new complexity for the medium prime case. In: Robshaw, M., Katz, J. (eds.) CRYPTO 2016, Part I. LNCS, vol. 9814, pp. 543-571. Springer, Heidelberg (Aug 2016). https: //doi.org/10.1007/978-3-662-53018-4_20

37. Kiyomura, Y., Inoue, A., Kawahara, Y., Yasuda, M., Takagi, T., Kobayashi, T.: Secure and efficient pairing at 256-bit security level. In: Gollmann, D., Miyaji, A., Kikuchi, H. (eds.) ACNS 17. LNCS, vol. 10355, pp. 59-79. Springer, Heidelberg (Jul 2017). https://doi.org/10.1007/978-3-319-61204-1_4 
38. Kleinjung, T., Aoki, K., Franke, J., Lenstra, A.K., Thomé, E., Bos, J.W., Gaudry, P., Kruppa, A., Montgomery, P.L., Osvik, D.A., te Riele, H.J.J., Timofeev, A., Zimmermann, P.: Factorization of a 768-bit RSA modulus. In: Rabin, T. (ed.) CRYPTO 2010. LNCS, vol. 6223, pp. 333-350. Springer, Heidelberg (Aug 2010). https://doi.org/10.1007/978-3-642-14623-7_18

39. Kleinjung, T., Diem, C., Lenstra, A.K., Priplata, C., Stahlke, C.: Computation of a 768-bit prime field discrete logarithm. In: Coron, J., Nielsen, J.B. (eds.) EUROCRYPT 2017, Part I. LNCS, vol. 10210, pp. 185-201. Springer, Heidelberg (Apr / May 2017). https://doi.org/10.1007/978-3-319-56620-7_7

40. Lewko, A.B.: Tools for simulating features of composite order bilinear groups in the prime order setting. In: Pointcheval, D., Johansson, T. (eds.) EUROCRYPT 2012. LNCS, vol. 7237, pp. 318-335. Springer, Heidelberg (Apr 2012). https://doi.org/ 10.1007/978-3-642-29011-4_20

41. Li, L., Wu, H., Zhang, F.: Pairing computation on edwards curves with high-degree twists. In: Lin, D., Xu, S., Yung, M. (eds.) Inscrypt. LNCS, vol. 8567, pp. 185-200. Springer, Guangzhou, China (November 27-30 2013). https://doi.org/10.1007/ 978-3-319-12087-4_12

42. Menezes, A., Sarkar, P., Singh, S.: Challenges with assessing the impact of NFS advances on the security of pairing-based cryptography. In: Phan, R.C., Yung, M. (eds.) Mycrypt. LNCS, vol. 10311, pp. 83-108. Springer, Kuala Lumpur, Malaysia (December 1-2 2016). https://doi.org/10.1007/978-3-319-61273-7_5

43. Montgomery, P.L.: Five, six, and seven-term Karatsuba-like formulae. IEEE Transactions on Computers 54, 362-369 (March 2005). https://doi.org/10.1109/TC. 2005.49

44. National Institute of Standards and Technology: Recommendation key management (part 1: General); SP 800-57 Part 1 (2016). https://doi.org/10.6028/NIST.SP. 800-57pt1r4, fourth revision

45. Pereira, G.C., Simplício, M.A., Naehrig, M., Barreto, P.S.: A family of implementation-friendly BN elliptic curves. Journal of Systems and Software 84(8), 1319 - 1326 (2011). https://doi.org/10.1016/j.jss.2011.03.083

46. Sarkar, P., Singh, S.: A general polynomial selection method and new asymptotic complexities for the tower number field sieve algorithm. In: Cheon, J.H., Takagi, T. (eds.) ASIACRYPT 2016, Part I. LNCS, vol. 10031, pp. 37-62. Springer, Heidelberg (Dec 2016). https://doi .org/10.1007/978-3-662-53887-6_2

47. Schirokauer, O.: The number field sieve for integers of low weight. Math. Comp. 79(269), 583-602 (January 2010). https://doi.org/10.1090/ S0025-5718-09-02198-X

48. Scott, M.: Implementing cryptographic pairings (invited talk). In: Takagi, T., Okamoto, T., Okamoto, E., Okamoto, T. (eds.) PAIRING 2007. LNCS, vol. 4575, pp. 177-196. Springer, Heidelberg (Jul 2007). https://doi.org/10. 1007/978-3-540-73489-5

49. Semaev, I.A.: Special prime numbers and discrete logs in finite prime fields. Math. Comp. 71(737), 363-377 (2002). https://doi.org/10.1090/ S0025-5718-00-01308-9

50. Sutherland, A.V.: Accelerating the CM method. LMS Journal of Computation and Mathematics 15, 172-204 (2012). https://doi.org/10.1112/S1461157012001015

51. Tanaka, S., Nakamula, K.: Constructing pairing-friendly elliptic curves using factorization of cyclotomic polynomials. In: Galbraith, S.D., Paterson, K.G. (eds.) PAIRING 2008. LNCS, vol. 5209, pp. 136-145. Springer, Heidelberg (Sep 2008). https://doi.org/10.1007/978-3-540-85538-5_10 
52. Vercauteren, F.: Optimal pairings. IEEE Transactions on Information Theory 56(1), 455-461 (Jan 2010). https://doi.org/10.1109/TIT.2009.2034881

53. Weber, D., Denny, T.F.: The solution of McCurley's discrete log challenge. In: Krawczyk, H. (ed.) CRYPTO'98. LNCS, vol. 1462, pp. 458-471. Springer, Heidelberg (Aug 1998). https://doi.org/10.1007/BFb0055747

54. Zhang, X., Lin, D.: Analysis of optimum pairing products at high security levels. In: Galbraith, S.D., Nandi, M. (eds.) INDOCRYPT 2012. LNCS, vol. 7668, pp. 412-430. Springer, Heidelberg (Dec 2012). https ://doi .org/10.1007/978-3-642-34931-7_ 24

\section{A Second part of the final exponentiation for $k=8$}

As an illustration, we give here pseudo-code that raises a finite field element $a$ to the power $c=\left(p+1-t_{0}\right) / r$, in the case $k=8$ and $i=1$ (code for all cases can be found in the code repository mentioned in $\S 1.1$ ). Recall that since the first part of the final exponentiation has been done, we know that $a^{p^{4}+1}=0$ so that $a^{-1}=a^{p^{4}}=\bar{a}$ where the conjugate is taken over the subfield $\mathbb{F}_{p^{4}}$. The formula below is specific to $i=1$, but we let $T=4 U+2 V$ which is the most general form (with $V \in\{0,1\}$ ). If we apply this to the parameters in $\S 6.1$, we can do some simplifications using $V=0$ (in square brackets below).

$$
\begin{array}{lr}
a_{y}=a^{y} ; a_{u}=a^{u} ; a_{Q}=a_{y}^{y} a_{u}^{u} ; b=a_{Q} \overline{a_{u}} ; & \left(2 \mathbf{c}_{u}+2 \mathbf{c}_{y}+2 \mathbf{m}_{k}\right) \\
b=b^{2} ; b=\left(b^{2} a\right)^{U} b^{V} ; b=b a_{y} ; & \left(\mathbf{c}_{T}+2 \mathbf{m}_{k}\right) \\
b=b^{2} ;\left[b=b a^{V}\right] ; b=\left(b^{2}\right)^{U} b^{V} ; b=b \overline{a_{y}} ; & \left(\mathbf{c}_{T}+\mathbf{m}_{k}\left[+\mathbf{m}_{k}\right]\right) \\
b=b^{2} ; b=\left(b^{2} a\right)^{U} b^{V} ; b=b a_{u} ; b=b \bar{a} ; & \left(\mathbf{c}_{T}+3 \mathbf{m}_{k}\right) \\
b=b^{2} ;\left[b=b a^{V}\right] ; b=\left(b^{2}\right)^{U} b^{V} ; b=b a_{Q} ; & \left(\mathbf{c}_{T}+\mathbf{m}_{k}\left[+\mathbf{m}_{k}\right]\right)
\end{array}
$$

The cost is $11 \mathbf{m}_{k}+4 \mathbf{c}_{T}+2 \mathbf{c}_{u}+2 \mathbf{c}_{y}$ in general, and $2 \mathbf{m}_{k}$ less if $V=0$, using the notations of $\S 5.2$. Here we use $\mathbf{c}_{T}$ to represent the cost of any set of operations whose cost is similar to $b=b^{2}$ followed by $b=\left(b^{2}\right)^{U} b^{V}$, although scheduling above is sometimes different.

\section{B Estimating the cost of NFS, NFS-HD and TNFS}

We would like to measure with the same methodology as Barbulescu and Duquesne in [7] the cost of computing a discrete logarithm in $\mathbb{F}_{p_{5}^{5}}, \mathbb{F}_{p_{6}^{6}}, \mathbb{F}_{p_{7}^{7}}$, and $\mathbb{F}_{p_{8}^{8}}$, and compare it with a prime field $\mathbb{F}_{p_{1}}$ of 3072 bits. In our setting the primes $p_{1}, p_{5}, p_{7}$ have no structure so we cannot use the Joux-Pierrot (JP) polynomial selection. We would like to show that the primes $p_{6}$ and $p_{8}$ have some structure but not enough to provide an advantage to the JP method (see §3). We compare the NFS, NFS-HD and TNFS variants. We give the best parameters we have found to minimise the running-time of the relation collection and the linear algebra steps, in the sense of [7]. Moreover we make available all the needed SageMath code to run our experiments (see $§ 1.1$ ) 
Contrary to [37], we not only estimate the size of the norms but we generate polynomials for each polynomial selection method available, we find parameters (see $\S$ B.2) so that enough relations are obtained, and we compare the estimated cost.

$A$ remark about Special NFS and TNFS. We consider that a prime $p$ in our set or primes $\left(p_{1}, p_{5}, p_{6}, p_{7}, p_{8}\right)$ is special and provides a notable advantage to the SNFS or STNFS algorithm if it can be written $p=P(u)$ where $u \approx p^{1 / d}$, and $P$ is a polynomial of degree at least 3 and whose coefficients are much smaller than $p^{1 / d}$. As a counterexample, for any prime $p$ we can use the base- $m$ polynomial selection method. It chooses $m=\left\lfloor p^{1 / d}\right\rceil$, writes $p$ in basis $m$ and outputs the corresponding polynomial $P$ such that $p=P(m)$. Then $\|P\|_{\infty}=m$, and the coefficients are large.

The vulnerabilities of pairing-friendly curves are the following:

- a special prime $p$ given by a polynomial of degree $>2$ and tiny coefficients, for instance $p(x)=36 x^{4}+36 x^{3}+24 x^{2}+6 x+1$ for BN curves [26, Ex. 6.8]. In that case, the Joux-Pierrot polynomial selection method (SNFS) allows a better complexity of NFS, in $L_{p^{k}}(1 / 3,1.923)$ instead of $L_{p^{k}}(1 / 3,2.201)$.

- a composite embedding degree $k$ allowing the Kim-Barbulescu variant of TNFS. Note that the original TNFS algorithm where $\operatorname{deg} h=k$ does apply but usually is not efficient when it is not combined with the Special variant.

- We note that the effectiveness of STNFS is not very clear. In [7], the authors found that for the KSS curves with $k=16$, the optimal choice is $\operatorname{deg} h=$ $k=16$, which is the original setting of Tower NFS, as in [9]. The key point is the special form of $p$ : the prime is given by a polynomial $p(s)=$ $\left(s^{10}+2 s^{9}+5 s^{8}+48 s^{6}+152 s^{5}+240 s^{4}+625 s^{2}+2398 s+3125\right) / 980$.

\section{B.1 Choices of polynomials}

Figure 11 shows the polynomials on the NFS setting and in the Tower-NFS setting.

Polynomial $\boldsymbol{h}$. In the Tower-NFS setting, the degree of the polynomial $h$ divides the degree $k$ of the extension. For $k=6$, we can take $\operatorname{deg} h \in\{2,3,6\}$ for example. We search for all monic polynomials $h$ of chosen degree, coefficients in $\{0,1,-1\}$ to minimise the norms, and of small Dedekind-zeta value $\zeta_{K_{h}}(2)$, so that its inverse $1 / \zeta_{K_{h}}(2)$ is as close as possible to 1 (in practice, we observed that this value is in the interval $] 0.4,1.0[$ ).

Polynomials $\boldsymbol{f}_{\mathbf{0}}, \boldsymbol{f}_{\mathbf{1}}$. These two polynomials are selected according to a polynomial selection method: JLSV 1 , JLSV 2 , Joux-Lercier, Generalised Joux-Lercier, Conjugation, Joux-Pierrot (Special case), Sarkar-Singh (see for example $[8,33,46]$ ). 


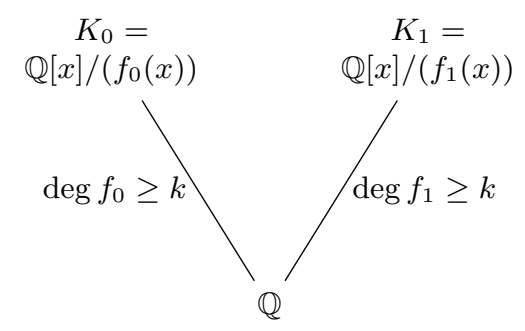

(a) NFS number fields, $p^{k} \mid \operatorname{Res}\left(f_{0}, f_{1}\right)$

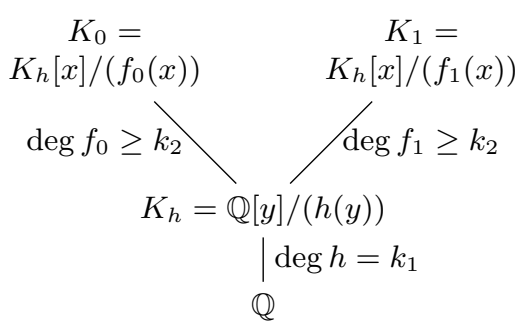

(b) Tower-NFS number fields, $k_{1} k_{2}=$ $k, p^{k} \mid \operatorname{Res}_{y}\left(h, \operatorname{Res}_{x}\left(f_{0}, f_{1}\right)\right)$

Fig. 11: Extensions of number fields for NFS and Tower variants

\section{B.2 Methodology for cost estimation}

Relation collection cost. To estimate this cost, we need first to discuss how relation collection will be performed. We make the conservative assumption that a sieving method can always be used. While this is of course commonplace for NFS computations, the same does not hold for TNFS, which needs $(2 \operatorname{deg} h$ )dimensional sieving for tuples of the form $\left(a_{0}, \ldots, a_{\operatorname{deg} h-1}, b_{0}, \ldots, b_{\operatorname{deg} h-1}\right)$. As a consequence of this assumption, the relation collection cost can be approximated as the size of the set of tuples. (Alternatively, relation collection can also use smoothness detection algorithms based on remainder trees, which can perform well in practice, see e.g. [39].)

We estimate the size of the set of tuples $\left(a_{0}, \ldots, a_{\operatorname{deg} h-1}, b_{0}, \ldots, b_{\operatorname{deg} h-1}\right)$ processed in the relation collection of the TNFS algorithm to be

$$
S_{\mathrm{TNFS}}^{0}(h, A)=(2 A+1)^{2 \operatorname{deg} h} / 2
$$

and its core part (duplicates removed) to be

$$
S_{\mathrm{TNFS}}^{1}(h, A)=(2 A+1)^{2 \operatorname{deg} h} /\left(2 w(h) \zeta_{K_{h}}(2)\right) .
$$

We consider that the cost of the relation collection is proportional to the first quantity $S_{\mathrm{TNFS}}^{0}(h, A)$, and to simplify, we assume that this is $S_{\mathrm{TNFS}}^{0}(h, A)$. We estimate that the number of unique relations obtained is $S_{\text {TNFS }}^{1}(h, A)$ times the average smoothness probability. For the NFS-HD algorithm, we estimate the size of the set of tuples $\left(a_{0}, \ldots, a_{\mathrm{dim}-1}\right)$ to be

$$
S_{\mathrm{NFS}-\mathrm{HD}}^{0}(\operatorname{dim}, A)=(2 A+1)^{\operatorname{dim}} / 2
$$

and its core part (duplicates removed) to be

$$
S_{\mathrm{NFS}-\mathrm{HD}}^{1}(\operatorname{dim}, A)=(2 A+1)^{\operatorname{dim}} /(2 \zeta(\operatorname{dim})) .
$$

Again, we consider that the cost of the relation collection is $S_{\mathrm{NFS}-\mathrm{HD}}^{0}(\operatorname{dim}, A)$, and the number of unique relations obtained is $S_{\mathrm{NFS}-\mathrm{HD}}^{1}(\operatorname{dim}, A)$ times the average smoothness probability. 
In the NFS algorithm, the elements in the relation collection are pairs of integers $(a, b)$. We need $a, b$ to be coprime: the probability is $1 / \zeta(2)=6 / \pi^{2} \approx 0.60$. For NFS-HD, the probability that a tuple of random integers $\left(a_{0}, \ldots, a_{\mathrm{dim}-1}\right)$ has gcd 1 is $1 / \zeta(\operatorname{dim})$. To avoid duplicates, the leading coefficient is chosen positive $((a, b)$ and $(-a,-b)$ give the same relation)

The generalisation to pairs of coprime ideals depends on the number field $K_{h}$ defined by $h$. The probability that two ideals of $K_{h}$ are coprime is $1 / \zeta_{K_{h}}(2)$. In practice we observed that it can vary from 0.44 to 0.99 . Then as in $[7, \S 5.2]$, we consider torsion units of $K_{h}$ (it happens if $h$ is a cyclotomic polynomial). Let $w$ be the index of $\{1,-1\}$ in the group of roots of unity in $K_{h}$. If $\mathfrak{q}$ is a prime ideal in $K_{f}$, then $u \mathfrak{q}$ is also a prime ideal giving the same relation, where $u$ is any root of unity of $K_{h}$. We can detect and avoid the case $u=-1$ but (up to now) there does not exist a way to avoid the other roots of unity. The number of tuples that will contribute to distinct relations is divided by $2 w$.

The non-torsion units do not contribute to duplicates: their coefficients being quite large, the coefficients of the ideal $u_{1} \mathfrak{q}$ overpass the bound $A$ and are not considered in the relation collection.

Average smoothness probability. To compute an average smoothness probability, we took at random $10^{6}$ coprime tuples $a$ of coefficients in $[-A, A]$ and positive leading coefficient (this requires about $\zeta(\operatorname{dim}) \cdot 10^{6}$ random tuples), resp. $10^{6}$ pairs of coprime ideals of $K_{h}$ (this requires about $\zeta_{K_{h}}(2) \cdot 10^{6}$ random tuples). Then we compute the resultants $N_{f}, N_{g}$ on both sides ( $f$ and $g$ ) and we compute the smoothness probability of that tuple as

$$
\operatorname{Pr}(a)=\operatorname{Pr}\left(N_{f} \text { is } B \text {-smooth }\right) \times \operatorname{Pr}\left(N_{g} \text { is } B \text {-smooth }\right) \text {. }
$$

We compute the average smoothness probability as the average over all the random unique tuples, that is $10^{-6} \sum_{\text {random } a \text {, coprime }} \operatorname{Pr}(a)$.

We estimate the smoothness probability on one side with the formula

$$
\operatorname{Pr}(N \text { is } B \text {-smooth }) \approx \delta(u)+(1-\gamma) \frac{\delta(u-1)}{\log N}, \text { where } u=\frac{\log N+\alpha}{\log B}
$$

where $\gamma \approx 0.577$ is Euler's constant, and $\delta$ is the Dickman rho function. ${ }^{6}$

Linear algebra cost (filtering, block-Wiedemann). We assume that the input of this step is a set of unique relations. Usually, a certain amount of excess is required: there are up to twice more relations than prime ideals involved in the relations (at this point, the matrix would be a vertical rectangle of twice more rows than columns). Before the linear algebra, the relations are processed to produce a dense matrix of good quality, in order to ease the linear algebra step. The filtering step removes the singletons (the prime ideals corresponding to

\footnotetext{
${ }^{6}$ We depart from the conventional notation $\rho$ for the Dickman rho function, to avoid confusion with $\rho=\log p / \log r$.
} 
columns that appear only in one relation). Doing this produces new singletons, so this step is done several times (two to ten times for example). Then a "clique removal" is performed, that also reduces part of the excess. Finally, a merge step increases the density of the matrix to some target density, reaching 125 to 200 non-zero entries per row in the recent record computations. The yield of the filtering step varies a lot in the literature: it reduced the size of the set of relations by a factor 9 for the SNFS-1024 DLP record [27], and by a factor 386 for the NFS-768 DLP record [39]

$$
c_{\text {filtering, } \min }=9, \quad c_{\text {filtering, } \max }=386 .
$$

We summarise in Table 12 the parameters of the filtering step for the recent record-breaking integer factorisations and discrete logarithm computations ${ }^{7,8}$. When we were not able to collect the data we put a question mark. Additional data for the DL-1024, DL-180, and DL-768 computations was collected from their respective authors (the cado-nfs-team and T. Kleinjung).The number of prime ideals is computed as log_integral (lpb0) + log_integral (lpb1). the PURGE step removes singletons, cliques, and for cado-nfs: removes the excess, for Kleinjung et al. records: removes part of the excess. the MERGE step increases the weight per row, reduces the number of rows and columns, and for Kleinjung et al. records: removes the final excess.

Contrary to [7], we propose a different interpretation of the filtering step yield: in our point of view, it is highly software-dependent and cryptanalyst-dependent. Indeed, the low values correspond to records by the cado-nfs team, while the high values correspond to Kleinjung et al. record computations (the software being not available in the latter case). At first glance, it seems to be due to software performance differences. To refine this impression, we decided to compare the two integer factorisation records of $2^{1039}-1$ and $2^{1061}-1$ by the SNFS algorithm: for $2^{1039}-1$, Kleinjung et al. have chosen a large prime bound of $2^{36}$ to $2^{38}$, while Childers has chosen the lower value $2^{33}$ for the larger integer $2^{1061}-1$ (Table 12). We can also compare the RSA-220 and RSA-768 record factorisations (220 and 232 decimal digits resp.) and obtain the same conclusion.

In fact, a strategy of oversieving was deployed for the DLP-768 record computation. The large prime bound was increased to $2^{36}$, while a bound of $2^{31}$ could have been enough (but it would have required a much higher effort in the linear algebra step). The ratio of ratios is $386.34 / 8.84=43.7$ and part of it is explained by the factor $2^{5}=32$ in the large prime bound choice. The larger set of relations to feed the filtering step allowed to obtain a matrix of better quality, reducing the linear algebra step. The density of rows seems more under control: from 134

\footnotetext{
${ }^{7}$ For the RSA-768 record factorisation, we used the corrected value $46.7 \mathrm{G}$ instead of $47.7 \mathrm{G}$, according to P. Zimmermann's webpage https://members.loria.fr/ PZimmermann/papers/\#rsa768.

${ }^{8}$ We mention that there was a typo in [7, Table 3]: in the factorisation of $2^{1039}-1$, there were $66.7 \mathrm{M}$ rows after filtering, not $82.8 \mathrm{M}$, and the reduction factor of the filtering step is 143 , not 167 .
} 
to 200 . We choose an upper bound: we assume that the density of a row is

$$
\text { weight per row }=200 \text {. }
$$

We estimate the time of the matrix-vector multiplications in the block-Wiedemann algorithm of the linear algebra step to be

$$
(\text { number of rows })^{2} \times \mathrm{w} \times(\text { weight per row })
$$

where $\mathrm{w}$ is the word-size of subgroup order (in our case, $\log _{2} r=256$ bits and

\begin{tabular}{|c|c|c|c|c|c|c|c|}
\hline & \multicolumn{3}{|c|}{ cado-nfs } & \multicolumn{3}{|c|}{ Kleinjung et al. } & NFS@HOME \\
\hline \begin{tabular}{|l|} 
record \\
references \\
year
\end{tabular} & $\begin{array}{c}\text { DL-1024 (S) } \\
{[27]} \\
2017\end{array}$ & \begin{tabular}{|c|} 
RSA-220 \\
{$[6]$} \\
2016
\end{tabular} & $\begin{array}{c}\text { DL-180 } \\
{[14]} \\
2014\end{array}$ & $\begin{array}{c}\text { DL-768 } \\
{[39]} \\
2017\end{array}$ & $\begin{array}{c}\text { RSA-768 } \\
{[38]} \\
2010\end{array}$ & $\begin{array}{c}2^{1039}-1(\mathrm{IF}) \\
{[1]} \\
2007\end{array}$ & $\begin{array}{c}2^{1061}-1(\mathrm{IF}) \\
{[17]} \\
2012\end{array}$ \\
\hline \multicolumn{8}{|l|}{ after sieving } \\
\hline $1 \mathrm{pb}$ & $2^{31}$ & $2^{34}$ & $2^{29}, 2^{30}$ & $2^{36}$ & $2^{37}$ to $2^{40}$ & $2^{36}$ to $2^{38}$ & $2^{33}$ \\
\hline unique rows $\left(R_{0}\right)$ & $248.94 \mathrm{M}$ & $1.17 \mathrm{G}$ & $179.29 \mathrm{M}$ & $9.08 \mathrm{G}$ & $46.76 \mathrm{G}$ & $13.8 \mathrm{G}$ & $671 \mathrm{M}$ \\
\hline prime ideals & $210.20 \mathrm{M}$ & $1.52 \mathrm{G}$ & $82.60 \mathrm{M}$ & $5.75 \mathrm{G}$ & 11.17 to $82.41 \mathrm{G}$ & 5.75 to $21.73 \mathrm{G}$ & $787 \mathrm{M}$ \\
\hline columns $\left(C_{0}\right)$ & $210.18 \mathrm{M}$ & $1.21 \mathrm{G}$ & $81.80 \mathrm{M}$ & $5.08 \mathrm{G}$ & $35.29 \mathrm{G}$ & $?$ & $?$ \\
\hline excess $\left(R_{0} / C_{0}\right)$ & 1.184 & 0.97 & 2.19 & 1.79 & 1.32 & & \\
\hline \multicolumn{8}{|l|}{ after rm singleton } \\
\hline rows $\left(R_{1}\right)$ & $222.77 \mathrm{M}$ & $613 \mathrm{M}$ & - & $7.84 \mathrm{G}$ & $24.62 \mathrm{G}$ & $?$ & $?$ \\
\hline columns $\left(C_{1}\right)$ & $173.58 \mathrm{M}$ & $594 \mathrm{M}$ & - & $\leq 3.77 \mathrm{G}$ & $\approx 15 \mathrm{G}$ & $?$ & $?$ \\
\hline excess $\left(R_{1} / C_{1}\right)$ & 1.28 & 1.03 & - & $\geq 2.07 \mathrm{G}$ & 1.64 & & \\
\hline ratio $R_{0} / R_{1}$ & 1.12 & 1.91 & - & 1.16 & 1.90 & & \\
\hline \multicolumn{8}{|l|}{ a. $\mathrm{rm}$ clique $+\operatorname{singl}$} \\
\hline rows $\left(R_{2}\right)$ & $95.93 \mathrm{M}$ & $496 \mathrm{M}$ & $21.46 \mathrm{M}$ & $1528 \mathrm{M}$ & $2.46 \mathrm{G}$ & $755.7 \mathrm{M}$ & $282 \mathrm{M}$ \\
\hline columns $\left(C_{2}\right)$ & $95.93 \mathrm{M}$ & $496 \mathrm{M}$ & $21.46 \mathrm{M}$ & $670 \mathrm{M}$ & $1.70 \mathrm{G}$ & $594.2 \mathrm{M}$ & $?$ \\
\hline excess $\left(R_{2} / C_{2}\right)$ & 1.00 & 1.00 & 1.00 & 2.28 & 1.45 & 1.27 & \\
\hline weight/row & 27.68 & 18.48 & 28.84 & $\leq 22.73$ & $?$ & $?$ & $?$ \\
\hline ratio $R_{0} / R_{2}$ & 2.595 & 2.36 & 8.40 & 5.94 & 19.02 & 18.26 & 2.38 \\
\hline \multicolumn{8}{|l|}{\begin{tabular}{|l|} 
after merge \\
\end{tabular}} \\
\hline rows $\left(R_{3}\right)$ & $28.15 \mathrm{M}$ & $132 \mathrm{M}$ & $7.29 \mathrm{M}$ & $23.5 \mathrm{M}$ & $192.80 \mathrm{M}$ & $66.7 \mathrm{M}$ & $90.3 \mathrm{M}$ \\
\hline columns $\left(C_{3}\right)$ & $28.15 \mathrm{M}$ & $132 \mathrm{M}$ & $7.29 \mathrm{M}$ & $23.5 \mathrm{M}$ & $192.80 \mathrm{M}$ & $66.7 \mathrm{M}$ & $90.3 \mathrm{M}$ \\
\hline weight/row & 200 & 175 & 150 & 134 & 144 & 143 & 125 \\
\hline ratio $R_{2} / R_{3}$ & 3.40 & 3.76 & 2.95 & 65.04 & 12.75 & 11.33 & 3.12 \\
\hline ratio $R_{0} / R_{3}$ & 8.84 & 8.86 & 24.60 & 386.34 & 242.55 & 207 & 7.43 \\
\hline
\end{tabular}
$\mathrm{w}=4$ words of 64 bits).

Table 12: Data from recent record computations.

\section{Comparison of (S)TNFS cost for curves with $k=6,8$}

In this section we explain how we obtained Figures 3 page 10 and 4 page 11. 
We estimate the cost of STNFS for three families of curves of respective embedding degree $k=6$ and $k=8$. To put a long story short, the important parameters in STNFS are the three polynomials $f_{0}, f_{1}, h$. The polynomial $h$ is chosen to define the first extension, its degree is a divisor of $k$. With $k=6$, we have $\operatorname{deg} h \in\{2,3,6\}$ and with $k=8$, then $\operatorname{deg} h \in\{2,4,8\}$; moreover $h$ is chosen with tiny coefficients, so that $\|h\|_{\infty}=1$ or 2 . The two other polynomials $f_{0}$ and $f_{1}$ are chosen such that $p^{k / \operatorname{deg} h} \mid \operatorname{Res}\left(f_{0}, f_{1}\right)$. One uses the Joux-Pierrot polynomial selection method to select them.

The relation collection step enumerates bivariate polynomials

$$
\boldsymbol{a}=\left(a_{0}+a_{1} y+\ldots+a_{7} y^{7}\right)+\left(b_{0}+b_{1} y+\ldots+b_{7} y^{7}\right) x:\left|a_{i j}\right| \leq A
$$

computes the integers $N_{f_{0}}=\left|\operatorname{Res}\left(\operatorname{Res}\left(\boldsymbol{a}, f_{0}\right), h\right)\right|, \quad N_{f_{1}}=\left|\operatorname{Res}\left(\operatorname{Res}\left(\boldsymbol{a}, f_{1}\right), h\right)\right|$ and factors these integers. The $B$-smooth integers produce a relation, where $B$ is the smoothness bound. The aim of setting the STNFS parameters is to find the optimal $A$ and $B$ associated to a triple of polynomials $\left(f_{0}, f_{1}, h\right)$. Without an implementation of STNFS, we can only simulate the parameters of the algorithm and estimate $A$ and $B$. The quantity to minimize for a faster running-time of STNFS is

$$
A^{(\operatorname{deg} h)\left(\operatorname{deg} f_{0}+\operatorname{deg} f_{1}\right)}\left\|f_{0}\right\|_{\infty}^{\operatorname{deg} h}\left\|f_{1}\right\|_{\infty}^{\operatorname{deg} h}
$$

under the constraint of collecting enough relations.

The BLS-6 curves have $\operatorname{deg} p=4$, while MNT-6 curves have $\operatorname{deg} p=2$. For a same bitlength of $p$, the seed $u$ is two times larger for MNT-6 curves. The TN-8 curves have $\operatorname{deg} p=6$, while FK-8 curves (with $D=4$ ) have $\operatorname{deg} p=8$. For polynomial selection in NFS, it implies that for the same bit length of $p$, the seed $u$ will be $33 \%$ smaller for the second curves. Since $u$ appears in the coefficients of the low degree polynomial $f_{1}$ in the Special setting, this second polynomial will produce smaller norms. On the contrary, the high degree polynomial $f_{0}$ will have a higher degree for the second curves and will produce larger norms. So the yield of Special-TNFS polynomials will be different for these two families. For our modified Cocks-Pinch, we have chosen to increase the coefficients of the polynomial $p(x)$ (that is, increasing $h_{t}$ and $h_{y}$ ) in order to increase $\left\|f_{0}\right\|_{\infty}^{\operatorname{deg} h}$ in equation (11).

\section{C.1 Comparison of curves with $k=6$}

We compare MNT6 and BLS6 curves [26, Theorem 5.2, Construction 6.6] to Cocks-Pinch $k=6$ curves (CP-6). The parameters are given in Table 13. The polynomials for simulating the Tower-NFS are given in Table 15. The graph of results is given in Figure 3 page 10.

\section{C.2 Comparison of curves with $k=8$}

We compare Tanaka-Nakamula (TN-8) curves [51], Fotiadis-Konstantinou (FK-8, $D=4$ ) curves $[24$, Table 3 row 4$]$ and our Cocks-Pinch $k=8$ curves (CP-8). The 


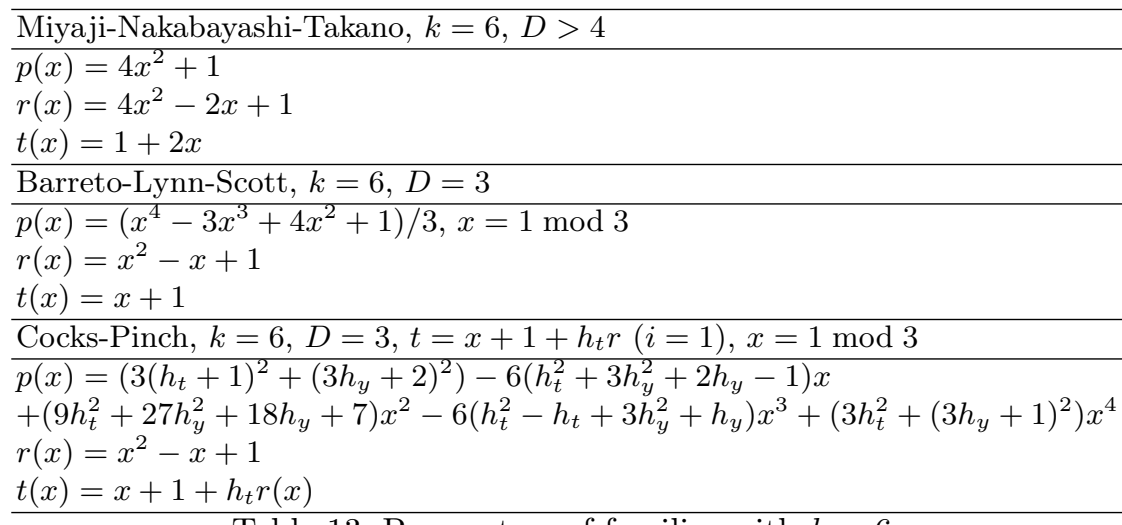

Table 13: Parameters of families with $k=6$.

\begin{tabular}{|c|c|c|c|c|c|}
\hline Curves & $p$ (bits) & $u$ (bits) & $r$ (bits) & $p^{k}$ (bits) & STNFS cost \\
\hline MNT-6 & 677 & 338 & 650 & 4061 & $2^{129}$ \\
\hline BLS-6 & 830 & 208 & 416 & 4980 & $2^{128}$ \\
\hline Cocks-Pinch-6 & 672 & 128 & 256 & 4028 & $2^{128}$ \\
\hline
\end{tabular}

Table 14: Parameter sizes for curves with $k=6$ to obtain 128 bits of security. We were not able to obtain MNT curve parameters with $p$ of exactly 672 bits but it would offer 128 bits of security as for Cocks-Pinch-6 curves.

parameters are given in Table 17. The polynomials for simulating the Tower-NFS are given in Table 19. The experimental data is summarized in Table 20. The graph of results is given in Figure 4 page 11. We conclude that to target a 128-bit security level (with some margin error), one needs a TN-8 curve with $p$ of 576 bits, or an FK-8 curve with $D=4$ and $p$ of 664 bits, or a Cocks-Pinch $k=8$ curve with $p$ of 544 bits. For each curve, the Miller loop length is the bit length of the seed $u$. This is (roughly) 96 bits for TN- 8 curves, 84 bits for FK- 8 curves, and 64 bits for our Cocks-Pinch curves. It means our Cocks-Pinch $k=8$ curves have a shorter Miller loop over a smaller prime field: it will be much faster. For the hard part of the final exponentiation, the advantage of Cocks-Pinch curves is less straightforward concerning its length, but the extension field is smaller anyway: $p^{k}$ has length 4352 bits for Cocks-Pinch curves, 4608 for TN curves and 5312 bits for FK curves. 


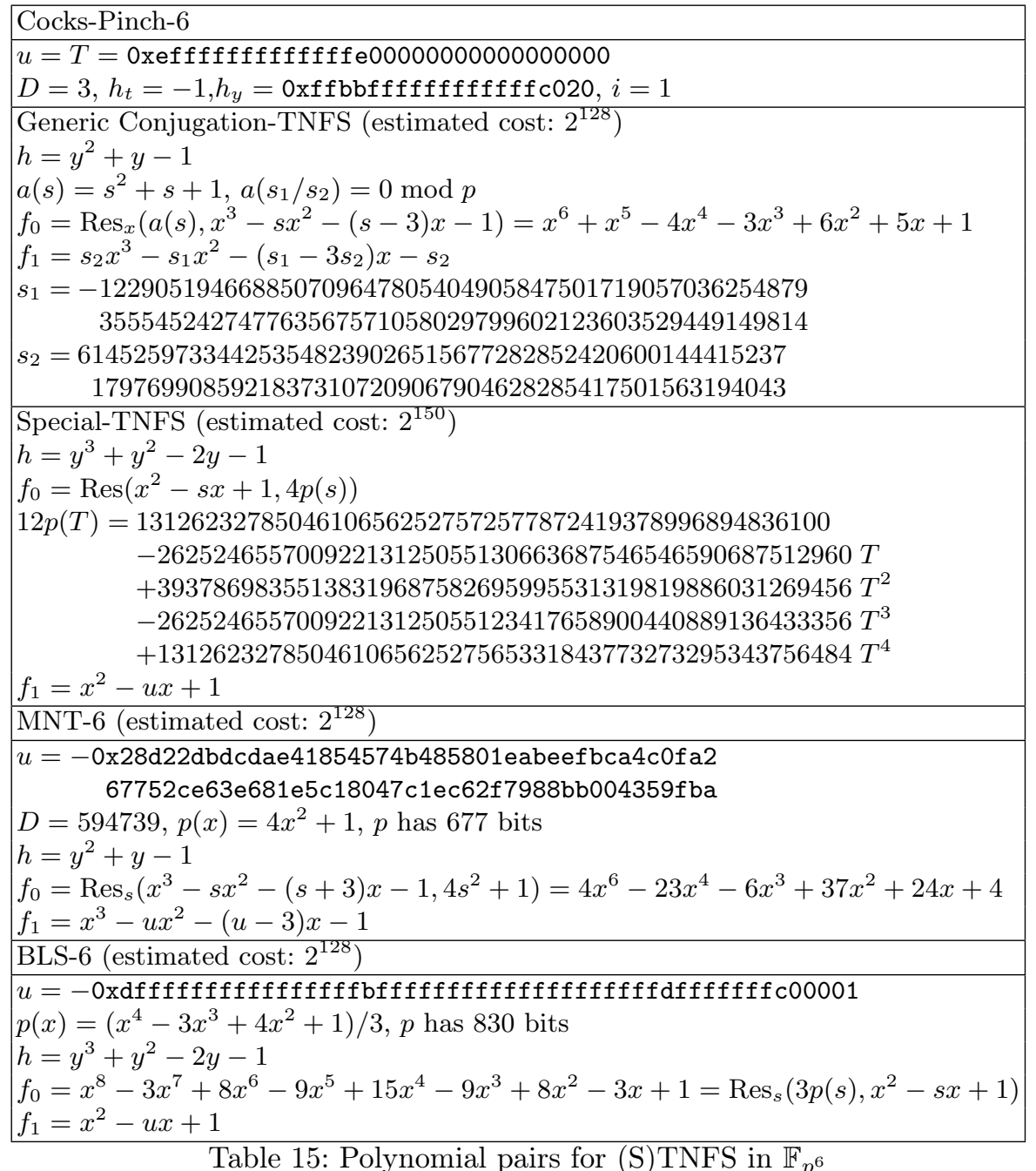




\begin{tabular}{|l||c||c||cc|}
\hline curve & BLS-6 & MNT-6 & \multicolumn{2}{|c|}{ Cocks-Pinch-6 modified } \\
\hline \hline$p$ (bits) & 830 & 677 & 672 & 672 \\
\hline$p^{k}$ (bits) & 4978 & 4061 & 4028 & 4028 \\
\hline$u$ (bits) & 208 & 338 & 128 & 128 \\
\hline polynomials & JP & JP & JP & Conj \\
\hline $\operatorname{deg} h$ & 3 & 2 & 3 & 2 \\
\hline $\operatorname{deg} f_{0}$ & 8 & 6 & 8 & 6 \\
\hline $\operatorname{deg} f_{1}$ & 2 & 3 & 2 & 3 \\
\hline$\left\|f_{0}\right\|_{\infty}$ & 15 & 37 & $\sim 2^{165}$ & 6 \\
\hline$\left\|f_{1}\right\|_{\infty}$ & $u \sim 2^{207}$ & $u \sim 2^{337}$ & $u \sim 2^{127}$ & $p^{1 / 2} \sim 2^{336}$ \\
\hline$A$ & 1185949 & 2858087529 & 14956378 & 2402798577 \\
\hline$B$ & $2^{65.926}$ & $2^{66.104}$ & $2^{77.569}$ & $2^{66.339}$ \\
\hline average $N_{f}($ bits) & 495.8 & 377.4 & 1067.1 & 370.6 \\
\hline average $N_{g}($ bits) & 741.5 & 858.0 & 523.7 & 853.9 \\
\hline$N_{f} \cdot N_{g}$ (bits) & 1237.2 & 1235.4 & 1590.8 & 1224.5 \\
\hline av. $B$-smooth. Pr & $2^{-64.12}$ & $2^{-66.02}$ & $2^{-74.41}$ & $2^{-64.63}$ \\
\hline rel. col. space & $2^{126.07}$ & $2^{128.65}$ & $2^{148.01}$ & $2^{127.648}$ \\
\hline factor basis & $2^{61.44}$ & $2^{61.62}$ & $2^{72.85}$ & $2^{61.85}$ \\
\hline rels. obtained & $2^{61.85}$ & $2^{62.41}$ & $2^{73.50}$ & $2^{62.80}$ \\
\hline total cost & $\mathbf{2}^{\mathbf{1 2 8}}$ & $\mathbf{2}^{\mathbf{1 2 9}}$ & $\mathbf{2}^{\mathbf{1 5 0}}$ & $\mathbf{2}^{\mathbf{1 2 8}}$ \\
\hline
\end{tabular}

Table 16: STNFS and TNFS parameters for curves with $k=6$.

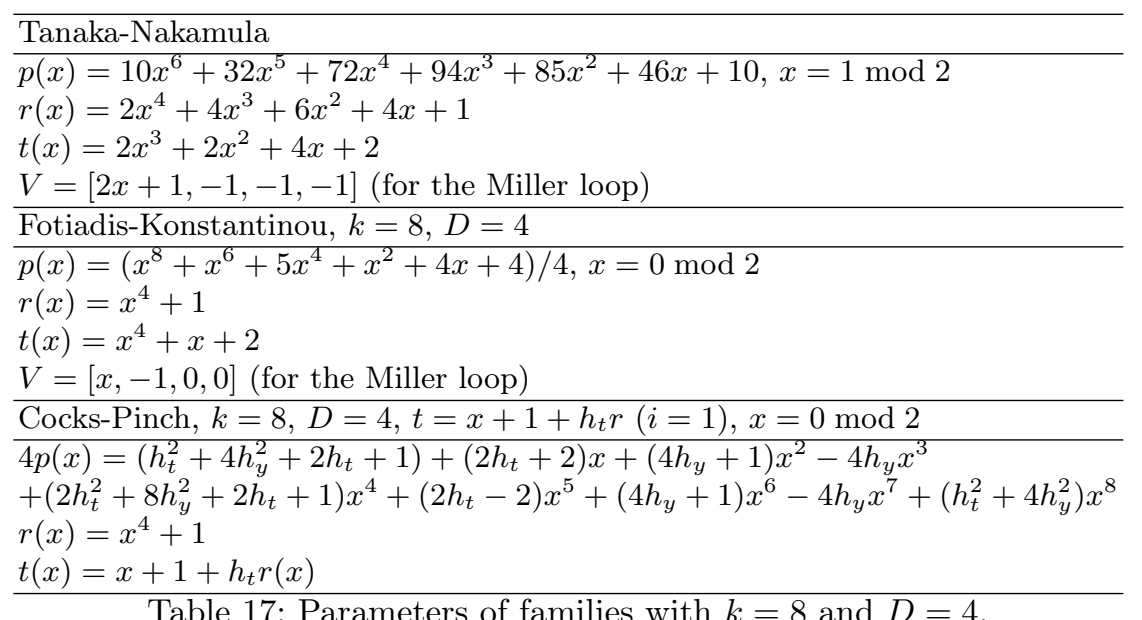

Table 17: Parameters of families with $k=8$ and $D=4$.

\begin{tabular}{|c|c|c|c|c|}
\hline Curves & $p$ (bits) & $u$ (bits) & $p^{k}$ (bits) & STNFS cost \\
\hline Tanaka-Nakamula & 576 & 96 & 4608 & $2^{128}$ \\
\hline Fotiadis-Konstantinou, $k=8, D=4$ & 664 & 84 & 5312 & $2^{128}$ \\
\hline Cocks-Pinch $k=8$ & 544 & 64 & 4352 & $2^{132}$ \\
\hline
\end{tabular}

Table 18: Parameter sizes for curves with $k=8$ to obtain 128 bits of security 


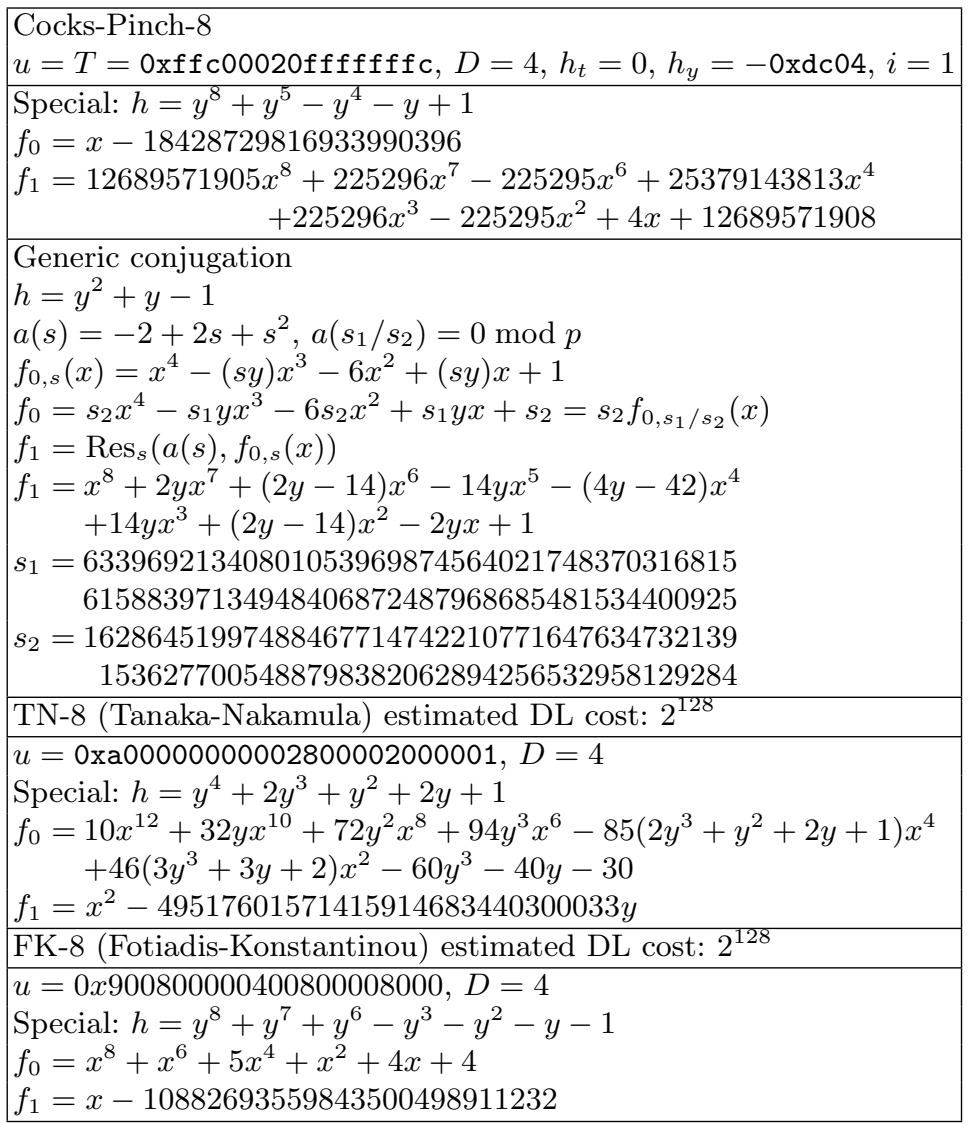

Table 19: Polynomial pairs for (S)TNFS 


\begin{tabular}{|l||c|c||cc|c||cc|}
\hline \multicolumn{1}{|l||}{ curve } & \multicolumn{2}{|c|}{ FK-8 } & \multicolumn{3}{c||}{ TN-8 } & Cocks-Pinch-8 modified \\
\hline \hline$p$ (bits) & 511 & 664 & 512 & 512 & 576 & 544 & 544 \\
\hline$p^{k}$ (bits) & 4081 & 5307 & 4093 & 4093 & 4603 & 4349 & 4349 \\
\hline$u$ (bits) & 65 & 84 & 85 & 85 & 96 & 64 & 64 \\
\hline polynomials & JP & JP & JP & JP & JP & JP & Conj \\
\hline $\operatorname{deg} h$ & 8 & 8 & 8 & 4 & 4 & 8 & 2 \\
\hline $\operatorname{deg} f_{0}$ & 8 & 8 & 6 & 12 & 12 & 8 & 8 \\
\hline $\operatorname{deg} f_{1}$ & 1 & 1 & 1 & 2 & 2 & 1 & 4 \\
\hline$\left\|f_{0}\right\|_{\infty}$ & 5 & 5 & 94 & 94 & 170 & 25379143813 & 42 \\
\hline$\left\|f_{1}\right\|_{\infty}$ & $u \sim 2^{64}$ & $u \sim 2^{83}$ & $u \sim 2^{84}$ & $u \sim 2^{84}$ & $u \sim 2^{95}$ & $u \sim 2^{64}$ & $\sim p^{1 / 2} \sim 2^{272}$ \\
\hline$A$ & 62 & 118 & 91 & 17995 & 30217 & 166 & 3646034133 \\
\hline$B$ & $2^{58.491}$ & $2^{66.039}$ & $2^{62.969}$ & $2^{62.969}$ & $2^{66.039}$ & $2^{70.427}$ & $2^{67.872}$ \\
\hline average $N_{f}$ (bits) & 448.6 & 505.7 & 384.9 & 711.7 & 752.8 & 797.5 & 504.4 \\
\hline average $N_{g}($ bits) & 562.7 & 722.7 & 732.7 & 449.9 & 499.8 & 574.0 & 791.3 \\
\hline$N_{f} \cdot N_{g}$ (bits) & 1011.3 & 1228.4 & 1117.6 & 1161.6 & 1252.6 & 1371.5 & 1295.7 \\
\hline av. $B$-smooth. Pr $\operatorname{Pr}$ & $2^{-55.74}$ & $2^{-61.29}$ & $2^{-59.81}$ & $2^{-63.91}$ & $2^{-62.98}$ & $2^{-67.04}$ & $2^{-65.82}$ \\
\hline rel. col. space & $2^{110.45}$ & $2^{125.22}$ & $2^{119.25}$ & $2^{120.08}$ & $2^{126.06}$ & $2^{133.07}$ & $2^{130.05}$ \\
\hline factor basis & $2^{54.19}$ & $2^{61.56}$ & $2^{58.56}$ & $2^{58.56}$ & $2^{61.56}$ & $2^{65.85}$ & $2^{63.34}$ \\
\hline rels. obtained & $2^{54.63}$ & $2^{62.18}$ & $2^{59.40}$ & $2^{58.69}$ & $2^{61.99}$ & $2^{65.95}$ & $2^{64.01}$ \\
\hline total cost & $\mathbf{2}^{113}$ & $\mathbf{2}^{\mathbf{1 2 8}}$ & $\mathbf{2}^{\mathbf{1 2 2}}$ & $\mathbf{2}^{\mathbf{1 2 2}}$ & $\mathbf{2}^{\mathbf{1 2 8}}$ & $\mathbf{2}^{136}$ & $\mathbf{2}^{\mathbf{1 3} 1}$ \\
\hline
\end{tabular}

Table 20: STNFS and TNFS parameters for curves with $k=8$ and $D=4$. 